\title{
Dimensionen und Ausprägungen fachinhaltlicher Kompetenz in den Naturwissenschaften - ein Systematisierungsversuch
}

\author{
Andreas Vorholzer ${ }^{1} \cdot$ Claudia von Aufschnaiter ${ }^{1}$ \\ Eingegangen: 14. März 2019 / Angenommen: 7. Januar 2020 / Online publiziert: 28. Januar 2020 \\ (c) Der/die Autor(en) 2020
}

\section{Zusammenfassung}

In den letzten ca. 15 Jahren haben die Modellierung und die Erfassung von Kompetenz im deutschen Sprachraum eine wichtige Rolle gespielt. Es wurde eine große Vielfalt von Kompetenzmodellen entwickelt, die auch dann unterschiedliche Dimensionen und Ausprägungen beschreiben, wenn sie auf den gleichen Kompetenzbereich Bezug nehmen. Ein zentrales Anliegen des Beitrages ist es, exemplarisch an einem kleinen Ausschnitt aus dem Forschungsfeld - der Modellierung fachinhaltlicher Kompetenz von Schüler*innen in den Naturwissenschaften - vorliegende Modelle (überwiegend aus dem deutschsprachigen Raum) aufeinander zu beziehen und dabei insbesondere die Varianz in den Dimensionierungen der Modelle herauszuarbeiten. Mithilfe induktiver Kategorienbildung wurden aus der Analyse bestehender Modelle sieben verschiedene Hauptdimensionen rekonstruiert, die im Beitrag erläutert werden: (1) Inhalt, (2) Kontext, (3) Vernetzung, (4) Denkprozess, (5) Abstraktion, (6) Angemessenheit und (7) Eigenständigkeit. Es wird diskutiert, welche Erträge diese systematische Perspektive für die Einordnung von Forschungsarbeiten im Bereich der Modellierung und Messung fachinhaltlicher Kompetenz hat und welche Herausforderungen und Fragestellungen sich aus dieser Perspektive für zukünftige Forschungsarbeiten ergeben. Dabei wird u. a. in den Blick genommen, inwiefern sich sowohl das methodische Vorgehen als auch die von uns rekonstruierten Dimensionen auf andere Bereiche naturwissenschaftlicher Kompetenz (z. B. Kompetenzbereich Erkenntnisgewinnung) und andere Zielgruppen (z.B. Studierende) übertragen lassen.

Schlüsselwörter Kompetenzmodellierung · Dimensionierung von Kompetenz · fachinhaltliche Kompetenz

Andreas Vorholzer

avorholzer@jlug.de

Claudia von Aufschnaiter

cvaufschnaiter@jlug.de

1 Institut für Didaktik der Physik, Justus-Liebig-Universität

Gießen, Karl-Glöckner-Str. 21C, 35394 Gießen, Deutschland 


\title{
Dimensions and Levels of Subject-matter Competency-An Attempt to Systematize Research in Science
}

\begin{abstract}
Within the last 15 years, modelling and assessing competence has been a major research area in German speaking countries. Research in this field has developed a wide range of competence models in which even models that aim to describe the same competence vary with respect to the dimensions and levels they comprise. In this paper, we report the analysis of research on modelling and assessing school students' subject-matter competency in science to illustrate the existing variety. By using an inductively developed system of categories, we have been able to extract seven dimensions from existing research which we describe in this paper: (1) content, (2) context, (3) interconnectedness, (4) cognitive process, (5) abstraction, (6) appropriateness, and (7) autonomy. We elaborate how our classification can be used to systematize empirical research on modelling and assessing subject-matter competency and discuss implications for future work in this field. In addition, we discuss to what extent our methodological approach and the dimensions we have identified may inform research on other areas of competence (e.g., competencies related to scientific practices, competencies of students at university level).
\end{abstract}

Keywords competence models $\cdot$ dimensions of competence $\cdot$ subject-matter competency

\section{Einleitung}

Die Modellierung und Erfassung von fachinhaltlicher Kompetenz (z. B. Kompetenzbereich Fachwissen) und von prozessbezogenen Kompetenzen (z.B. Kompetenzbereiche Erkenntnisgewinnung, Kommunikation, Bewertung; siehe z.B. KMK 2005a, 2005b, 2005c) ist seit über 15 Jahren ein wichtiges Feld nationaler fachdidaktischer und psychologischer Forschung. ${ }^{1}$ In dieser Forschung sind vielfältige Modelle entstanden, die sich selbst dann deutlich unterscheiden, wenn auf gleiche Kompetenzen und Zielgruppen Bezug genommen wird (für fachinhaltliche Kompetenz in den Naturwissenschaften vgl. z.B. die Übersicht in Parchmann 2010; von Aufschnaiter und Rogge 2010). Die Vielfalt der Modelle zu einer Kompetenz ermöglicht zwar facettenreiche Zugriffe auf das Konstrukt, erschwert aber gleichzeitig die Einordnung und wechselseitige Bezugnahme von Befunden, weil Unterschiede in den Modellierungen nicht immer leicht erkennbar sind. Dies Schwierigkeiten treten z. B. dann auf, wenn gleiche Begrifflichkeiten mit unterschiedlichen Bedeutungen belegt werden. Darüber hinaus kann erst in der zusammenfassenden Bezugnahme von Modellen, die auf die gleiche Kompetenz (und Zielgruppe) gerichtet sind, deutlich werden, worauf in der Konstruktion von Aufgaben zur Kompetenzmessung geachtet werden sollte, um zu interpretierbaren Ergebnissen zu gelangen.

Ziel dieses Beitrags ist es, die Varianz in Modellen zu einem Konstrukt herauszuarbeiten und in eine Systematik zu überführen, um eine Orientierung für zukünftige For-

\footnotetext{
${ }^{1}$ U.a. im Rahmen des Schwerpunktprogrammes „Kompetenzmodelle zur Erfassung individueller Lernergebnisse und zur Bilanzierung von Bildungsprozessen" der Deutschen Forschungsgemeinschaft (Laufzeit 2007-2013) sowie im vom Bundesministerium für Bildung und Forschung geförderten Programm „Kompetenzmodelle und Instrumente der Kompetenzerfassung im Hochschulsektor“ (Laufzeit 2011-2019).
}

schungsarbeiten zu schaffen. Wir beziehen uns dabei auf das in den Naturwissenschaften sehr gut untersuchte Feld der fachinhaltlichen Kompetenz und beschränken uns darin auf Modelle zur Beschreibung der Kompetenz von Schüler*innen. In dieser Beschränkung, sowohl im Hinblick auf die Kompetenz als auch mit Blick auf die Zielgruppe, schaffen wir einen vergleichbaren Rahmen, in dem Varianz kein Artefakt eines unterschiedlichen inhaltlichen Zugriffs oder unterschiedlicher adressierter Zielgruppen ist.

Ausgangspunkt unserer Überlegungen bildet der Artikel von Schecker und Parchmann (2006), in dem der damalige Stand der naturwissenschaftsbezogenen Kompetenzforschung aufgearbeitet wird. Die Autor*innen gehen darin der Trennung zwischen normativen und deskriptiven Kompetenzmodellen sowie der Unterscheidung von Struktur- und Entwicklungsmodellen nach. In ihrer Diskussion der Modellierungen befassen sie sich zudem kritisch mit Fragen der Dimensionierung und Graduierung naturwissenschaftlicher Kompetenz und formulieren Desiderata für zukünftige Kompetenzforschung. Wir nehmen die bisher entwickelten Kompetenzmodelle zum Anlass, an die von Schecker und Parchmann (2006) angestoßenen Überlegungen in zweifacher Weise anzuknüpfen:

1. Wir analysieren gängige Modellierungen fachinhaltlicher Kompetenz, um herauszuarbeiten, welche unterschiedlichen Dimensionen und Zuschnitte innerhalb dieser Dimensionen zur Modellierung von Kompetenz genutzt werden (können). Wir unterscheiden im Vergleich der Modelle überwiegend nicht zwischen normativen und deskriptiven Modellen, weil die Überlegungen zur Dimensionierung von Kompetenz zunächst unabhängig davon sind, ob es sich um theoretisch abgeleitete oder aus empirischen Daten konstruierte Modelle handelt. 


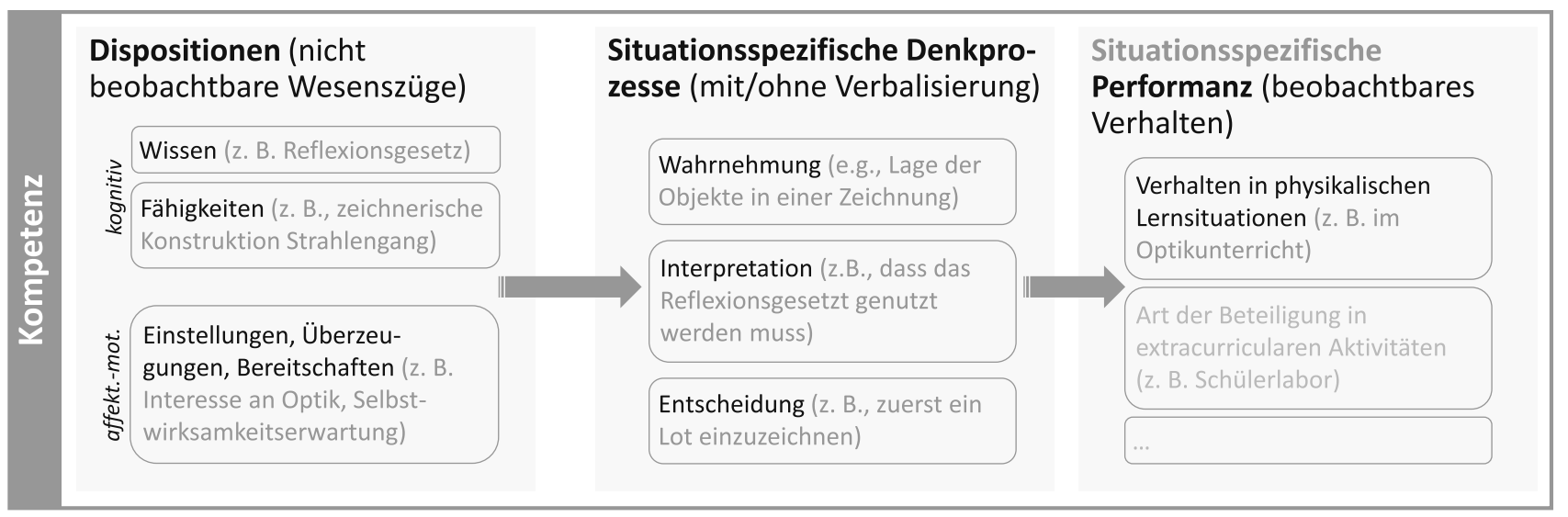

Abb. 1 Kompetenzmodell in Anlehnung an Blömeke et al. (2015, S. 7) mit naturwissenschaftsbezogenem Beispiel

2. Wir entwerfen eine Systematik zur Klassifikation von in Modellierungen fachinhaltlicher Kompetenz beschriebenen Dimensionen, um der Frage nach der Anzahl und Untergliederung von nicht-stufbaren und potenziell stufbaren Dimensionen (vgl. Schecker und Parchmann 2006, S. $53 \mathrm{ff}$.) von Kompetenz nachzugehen.

Die aus (1) und (2) abgeleitete Systematik liefert auf der einen Seite wichtige Hinweise für die variablenkontrollierte Erfassung von Kompetenz und die präzise Formulierung von Kompetenzbeschreibungen (vgl. Abschn. 5). Auf der anderen Seite bildet die systematische Darstellung der gegenwärtig für die Modellierung fachinhaltlicher Kompetenz von Schüler*innen genutzten Dimensionen und Ausprägungen einen Ausgangspunkt für die Diskussion fachdidaktischer Fragen der Kompetenzmodellierung. Mit Blick auf das Spannungsfeld zwischen der Differenziertheit auf der einen und der Handhabbarkeit eines Modells auf der anderen Seite gehören dazu u. a. die Frage nach der Relevanz einzelner Dimensionen sowie die Frage nach der (empirischen) Trennbarkeit von Dimensionen (vgl. Abschn. 5). Ausgehend von der Systematik kann zudem diskutiert werden, inwiefern sich die identifizierten Dimensionen auch zur Beschreibung von Kompetenz in anderen Bereichen (z.B. Erkenntnisgewinnung) oder für andere Zielgruppen (z.B. Studierende) eignen (vgl. Abschn. 6).

\section{Begriffliche Fassung von Kompetenz}

Es herrscht große Einigkeit, dass es sich bei Kompetenzen um erlernbare, domänenspezifische kognitive sowie motivationale, volitionale und soziale Fähigkeiten, Fertigkeiten und Bereitschaften handelt (vgl. Kompetenzbegriff nach Weinert 2001; diskutiert in z. B. Hartig und Klieme 2006; Klieme et al. 2007). Konsens ist auch, dass das Kompetenzkonstrukt von allgemeinen (fachunspezifischen) Kon- strukten wie Intelligenz und Schlüsselqualifikationen abzugrenzen ist (z.B. Hartig und Klieme 2006). Obwohl in schulischen Kontexten gelegentlich missverstanden, scheint zudem unstrittig, dass „Wissen“ im Sinne einer verständnisvollen Nutzung von Fakten, Definitionen, Regeln, Gesetzen und Theorien, ebenfalls Bestandteil von Kompetenz ist (Klieme et al. 2007; von Aufschnaiter und Hofmann 2014; Vorholzer, von Aufschnaiter und Kirschner 2016; Weinert 2000).

In dem von Weinert (2001) geprägten Kompetenzbegriff können drei deutlich unterschiedliche Bereiche identifiziert werden, die Bestandteile naturwissenschaftlicher Kompetenz sind bzw. sein müssten: Während der kognitive Bereich u. a. fachbezogene Kenntnisse, Fähigkeiten und Fertigkeiten umfasst, werden mit dem motivational-volitionalen und dem sozialen Bereich Kenntnisse, Fähigkeiten und Bereitschaften adressiert (z. B. die fachbezogenen Interessen und Selbstwirksamkeitserwartungen). Alle drei Bereiche lassen sich so deuten, dass es sich um domänenspezifische „Dispositionen“ (vgl. Blömeke et al. 2015) im Sinne von Personenmerkmalen handelt, die in der kognitiven Struktur verankert und durch Lernen veränderbar sind. Insbesondere neuere Arbeiten gehen davon aus, dass Kompetenz nicht alleine mit Personenmerkmalen gleichzusetzen ist bzw. gleichgesetzt werden sollte. Blömeke et al. (2015) betonen, dass sich Kompetenz aus (situationsübergreifenden) dispositionellen Anteilen (Abb. 1 links) und (situationsspezifischen) performatorischen Anteilen (Abb. 1 rechts) zusammensetzt. Blömeke et al. formulieren zudem situationsspezifische Denkprozesse (Abb. 1 Mitte), die im Sinne eines vermittelnden Elements zwischen Dispositionen und Performanz gedeutet werden können und damit ebenfalls einen Bestandteil von Kompetenz bilden.

Das in Abb. 1 dargestellte Modell ist für die Dispositionen an andere Modellierungen (z.B. Weinert 2001) anschlussfähig, da es alle kognitiven, motivational-volitionalen und sozialen Kenntnisse, Fähigkeiten und Bereitschaf- 
ten umfasst, über die ein Individuum verfügt (bei Blömeke et al. 2015, S. 7 als „Cognition“ und „Affect-motivation“ bezeichnet). Für den Bereich der Performanz lässt das Modell aber unterschiedliche Auslegungen zu, die trotz des Rückgriffs auf den gleichen theoretischen Rahmen auf erkennbar unterschiedliche Verständnisse von Kompetenz verweisen. Unter der Annahme, dass es sich bei Dispositionen um latente Variablen handelt, die immer nur über die Erhebung manifester Variablen (z. B. Lösungen zu Testaufgaben, Aussagen in Interviews, Verhaltensbeobachtungen) empirisch messbar werden, lässt sich Performanz als manifeste Variable deuten. Dieses Verständnis von Performanz ist nach unserer Einschätzung jedoch nicht deckungsgleich mit Performanz im Sinne des Modells von Blömeke et al. (2015). Im Sinne des Modells wäre ein Fokus auf Kompetenz aus der Perspektive der Performanz alleine auf die Kompetenzschätzung auf der Basis der Güte der Performanz gerichtet. Es würden dabei jedoch keine Aussagen über die Dispositionen oder Denkprozesse abgeleitet, die diese Performanz hervorgebracht haben könnten. Ein Beispiel für einen solchen Zugang liefern Kulgemeyer und Schecker (2013), die Erklärungen von Schüler*innen analysieren, darin aber nicht rekonstruieren, über welche Kenntnisse zum Erklären die Schüler*innen verfügen (müssten). Welches Verständnis von Kompetenz zugrunde gelegt wird - Kompetenz als latente mit Performanz als manifester Variablen versus Performanz als eigenständiger Aspekt von Kompetenz -, wird nicht immer deutlich. Insbesondere kann eine identische Formulierung einzelner Kompetenzen vorliegen, diese aber einmal als mental verankerte Kenntnisse und Fähigkeiten (Dispositionen) und ein anderes Mal als sichtbare Verhaltensweisen (Performanz) interpretiert werden.

In der Zusammensetzung aus spezifischen Bereichen (kognitiv, motivational-volitional, sozial) sowie dispositionellen, performatorischen und prozesshaften Anteilen wird die Komplexität eines (aktuellen) Kompetenzbegriffs deutlich, dem sich Forschung immer nur in Auszügen nähern kann. ${ }^{2}$ Selbst in einer solchen Näherung bleiben die Modellierungen komplex und deutlich variantenreich, was wir im weiteren Verlauf des Beitrags exemplarisch am Beispiel fachinhaltlicher Kompetenz (Wissen, Fähigkeiten und Fertigkeiten) als einem (Teil-)Bereich der kognitiven Dispositionen (Abb. 1 links) illustrieren werden. Wir beschränken uns dabei auf Modelle, die sich auf Schüler*innen beziehen.

\footnotetext{
${ }^{2}$ Diese Beschränkung ist typisch für alle Modelle und nicht per se problematisch (siehe Abschn. 4). Vielmehr erfordert sie eine kritische Diskussion dieser Beschränkung, auch im Sinne der Diskussion der Validität der vor dem Hintergrund einer spezifischen Modellierung gewonnenen Erkenntnisse (vgl. Messick 1995).
}

\section{Vorgehen zur Entwicklung einer Systematik}

\section{Einordnung und Abgrenzung fachinhaltlicher Kompetenz}

Ein erster Schritt zur Entwicklung eines systematischen Überblicks ist die Eingrenzung fachinhaltlicher Kompetenz als Teilbereich fachlicher, naturwissenschaftlicher Kompetenz (vgl. Übersicht in Tab. 1). Wir haben uns hier an den deutschen Bildungsstandards orientiert, in denen fachinhaltliche Kompetenz im Kompetenzbereich „Fachwissen“ ausgewiesen und darin klar als kognitiver Aspekt von Kompetenz aufgefasst sowie konsequent von anderen fachbezogenen Kompetenzen (,prozessbezogene Kompetenzbereiche": Erkenntnisgewinnung, Kommunikation und Bewertung) abgegrenzt wird. Anschließend wurde eine Literaturrecherche im deutschsprachigen Raum durchgeführt, deren Gegenstand Publikationen zur Modellierung und Beschreibung der Struktur oder Entwicklung von fachinhaltlicher Kompetenz (im oben genannten Sinne) war (siehe Übersicht in Tab. 1). Die Fokussierung auf den deutschsprachigen Raum erschien uns nicht nur aufgrund des gewählten Begriffsverständnisses sinnvoll, sondern auch, weil die Forschung zu Kompetenzmodellierung und -messung aus unserer Sicht hier einen deutlichen Schwerpunkt hat. Um die Anschlussfähigkeit der im folgenden dargestellten Überlegungen an den internationalen Diskussionsstand aufzuzeigen, werden bei der Betrachtung der einzelnen Dimensionen auch die US-amerikanischen Next Generation Science Standards (NGSS; NGSS Lead States 2013; s. a. NRC 2012), die im deutschen Sprachraum immer wieder als Bezugspunkt genannt werden (z. B. Koenen et al. 2017; Schecker et al. 2016; Schwichow und Nehring 2018) sowie das Schweizer HarmoS-Kompetenzmodell (EDK 2011; Ramseier et al. 2011) und die Modellierungen aus internationalen Vergleichsstudien wie TIMSS (Baumert et al. 1998, 2000) und PISA (OECD 2006, 2017) miteinbezogen. Die Analyse umfasst sowohl Modelle, die fachinhaltliche Kompetenz für ein spezifisches Fach (Biologie, Chemie oder Physik; z. B. KMK 2005a, 2005b, 2005c) beschreiben, als auch Modelle, die naturwissenschaftsübergreifende Beschreibungen vornehmen (z. B. EDK 2011). Eine Fokussierung auf ein bestimmtes Fach erscheint uns hingegen nicht erforderlich, da auf der einen Seite nicht alle Modellierungen systematisch zwischen den Fächern trennen und auf der anderen Seite anzunehmen ist, dass sich die rekonstruierten Dimensionen fachübergreifend in den Naturwissenschaften anwenden lassen.

Nach einer ersten Sichtung der Modelle haben wir uns in einem diskursiven Verfahren in der Autorengruppe darauf verständigt, welche Bereiche in jedem Modell dem Bereich fachinhaltlicher Kompetenz zuzuordnen sind (in Tab. 1 mit einem hochgestellten ,a“ gekennzeichnet) und 
Tab. 1 Überblick über die analysierten Kompetenzmodelle und die in diesen Modellen vorgenommenen Zuschnitte fachlicher Kompetenz

Kompetenzmodell
KMK (2005a, 2005b, 2005c): Bildungsstan-
dards für die Fächer Biologie, Chemie un
Physik
EDK (2011): Harmonisierung der obli-
gatorischen Schule, Kompetenzmodell
Naturwissenschaften (HarmoS Naturwis-
senschaften)
senschaften)

NGSS Lead States (2013): Next Generation Science Standards (NGSS)

OECD (2017): PISA 2015 Framework

Baumert et al. (1998, 2000): Rahmenmodell TIMSS II/III

Kauertz et al. (2010): Kompetenzmodell zur Evaluation der Standards in den naturwissenschaftlichen Fächern der Sekundarstufe I (ESNaS)

Kleickmann et al. (2010): Modell naturwissenschaftlicher Kompetenz im Grundschulalter

Bernholt et al. (2009): Modell der hierarchischen Komplexität Chemie (MHC-C)

Neumann et al. (2007): Modell physikalischer Kompetenz

Neumann et al. (2010): Entwicklungsmodell physikalischer Kompetenz

Schecker und Parchmann (2006): Rahmenmodell für Kompetenzstrukturen

von Aufschnaiter und Rogge (2010): Modellierung der Verläufe der Kompetenzentwicklung (Fachwissen)

kursiv Benennung des Bereichs im jeweiligen Modell

${ }^{a}$ Teilbereich, in dem fachinhaltliche Kompetenz beschrieben wird und der in die weiterführenden Analysen einbezogen wurden
Zuschnitt fachlicher Kompetenz

Kompetenzbereiche

Fachwissen $^{\mathrm{a}}$

Erkenntnisgewinnung

Kommunikation

Bewertung

Handlungsaspekte

Interesse und Neugierde wecken

Fragen und untersuchen

Informationen erschließen ${ }^{\mathrm{a}}$

Ordnen, strukturieren, modellieren ${ }^{\mathrm{a}}$

Einschätzen und beurteilen

Entwickeln und umsetzen

Mitteilen und austauschen ${ }^{\mathrm{a}}$

Eigenständig arbeiten

Practices

Asking Questions and Defining Problems

Developing and Using Models

Planning and Carrying Out Investigations

Analyzing and Interpreting Data

Using Mathematics and Computational Thinking

Constructing Explanations and Designing Solutions ${ }^{\mathrm{a}}$

Engaging in Argument from Evidence

Obtaining, Evaluating, and Communicating Information

Scientific Competencies

Phänomene naturwissenschaftlich erklären ${ }^{\mathrm{a}}$

Naturwissenschaftliche Forschung bewerten und Untersuchungen planen

Daten und Evidenz naturwissenschaftlich interpretieren

Stoffgebiete

Geographie und Biowissenschaften ${ }^{\mathrm{a}}$

Biologie und Biowissenschaften ${ }^{\mathrm{a}}$

Physik $^{\mathrm{a}}$

Technologie ${ }^{\mathrm{a}}$

Naturwissenschaftliches Arbeiten und Argumentieren

Kompetenzbereiche der KMK Bildungsstandards

Bereiche

Naturwissenschaftliches Wissen ${ }^{\mathrm{a}}$

Wissen über Naturwissenschaften

Es wird nur der Bereich Fachwissen ${ }^{\mathrm{a}}$ (im Sinne der Bildungsstandards) modelliert

Es wird nur der Bereich Fachwissen ${ }^{\mathrm{a}}$ (im Sinne der Bildungsstandards) modelliert

Es wird nur der Bereich Fachwissen ${ }^{\mathrm{a}}$ (im Sinne der Bildungsstandards) modelliert

Kompetenzbereiche der KMK Bildungsstandards

Es wird nur der Bereich Fachwissen ${ }^{\mathrm{a}}$ (im Sinne der Bildungsstandards) modelliert 
somit in die weiteren Analysen einbezogen werden. Typischerweise konnte auch in Modellen, die nicht direkt auf die nationalen Bildungsstandards bzw. Kompetenzbereich Fachwissen Bezug nehmen, ein fachinhaltlicher Bereich identifiziert werden. So unterscheidet beispielsweise das PISA-Framework (OECD 2017; s.a. Tab. 1) in ähnlicher Weise wie die Bildungsstandards zwischen fachinhaltlichen Kompetenzen (,naturwissenschaftliche Phänomene erklären“) und prozessbezogenen Kompetenzen (bewerten, planen, interpretieren). Vereinzelt war für uns eine klare Abgrenzung von fachinhaltlichen gegenüber prozessbezogenen Kompetenzen nicht erkennbar. Ein Beispiel hierfür ist das Schweizer HarmoS-Modell, in dem acht „Handlungsaspekte“ beschrieben werden (vgl. Tab. 1), die sowohl fachinhaltliche und prozessbezogenen als auch kognitive und motivational-volitionale Dispositionen umfassen (z. B. der Handlungsaspekt „Interesse und Neugierde“). Bei der Auswahl der Bereiche haben wir in diesen Fällen für die weiteren Analysen alle Bereiche berücksichtigt, die zumindest in Teilen auf fachinhaltliche Kompetenz Bezug nehmen (z. B. „Informationen erschließen“ oder „Mitteilen und austauschen“ im HarmoS-Modell; vgl. Tab. 1).

\section{Literaturrecherche und induktive Kategorienbildung}

Für alle ausgewählten Modelle wurde jede zur Beschreibung fachinhaltlicher Kompetenz genutzte Dimension und deren Ausprägungen separat notiert und in einem induktiven Verfahren zu Dimensionen kategorisiert (induktive Kategorienbildung in Anlehnung an Mayring und Fenzl 2014). Dabei wurden nach und nach so lange neue Dimensionen als Kategorien gebildet, bis eine Sättigung eingetreten ist, sich also jede weitere in einem der gesichteten Modelle beschriebene Dimension in eine bereits bestehende Kategorie einsortieren ließ. Die in Abschn. 4 beschriebenen Systematik ist daher nicht in dem Sinne vollständig, dass sie alle publizierten Modelle fachinhaltlicher Kompetenz beinhaltet, sondern soll lediglich alle in diesen Modellen beschriebenen Dimensionen abbilden. Es ist uns hierbei wichtig zu betonen, dass wir bei der Klassifikation der Dimensionen versucht haben, mit Hilfe der jeweils zugehörigen Beschreibung zu rekonstruieren, welche Facette von Kompetenz aus Sicht der Autor*innen, die das Modell entwickelt haben, vermutlich jeweils abgebildet werden soll. Der erste Analyseschritt der induktiven Kategorienbildung und der (ersten) Zuweisung von in der Literatur beschriebenen Dimensionen wurde von den beiden Mitgliedern der Autorengruppe unabhängig voneinander durchgeführt. Die finale $\mathrm{Zu}$ weisung der gesichteten Dimensionen zu einer Kategorie erfolgte anschließend in einem diskursiven Konsensverfahren. Dieser Interpretationsschritt hat zur Folge, dass die in einzelnen Modellen identisch oder sehr ähnlich bezeichneten Dimensionen oder Ausprägungen z.T. unterschied- lichen Kategorien zugewiesen wurden, da sie aus unserer Sicht unterschiedliche Facetten von Kompetenz modellieren (z. B. „Kognitive Aktivität“ in Tab. 3). Wir haben uns aufgrund des interpretatorischen Prozesses und den z.T. nur knappen Beschreibungen der Dimensionen in den zugehörigen Veröffentlichungen bewusst für ein Verfahren der Konsensbildung entschieden, um sicherzustellen, dass wir alle vorliegenden Dimensionen in unserer Klassifikation sinnvoll erfassen. Mit Blick auf die entstandene Systematik ist dabei nicht zentral, ob sich alle in den Modellen beschriebenen Dimensionen eindeutig einer bestimmten Kategorie zuordnen lassen, sondern nur, dass keine zusätzliche Kategorie entsteht.

In Anlehnung an die Grundidee von Kompetenzmodellen bezeichnen wir die von uns gebildeten Kategorien im Folgenden als Dimension bzw. Subdimension. Wir weisen in den Tab. 2 und 3 exemplarisch Bereiche aus publizierten Kompetenzmodellen den von uns konstruierten (Sub-)Dimensionen zu. Um begriffliche Unklarheiten zu vermeiden, möchten wir an dieser Stelle darauf hinweisen, dass alles, was wir im Folgenden als Bereich eines Modells bezeichnen, in den analysierten Modellen selbst typischerweise als Dimension bezeichnet wird.

\section{Vorschlag einer Systematik für Dimensionen fachinhaltlicher Kompetenz}

Einen ersten Zugang zur Systematisierung der in der Literatur identifizierten Dimensionen stellt die Unterscheidung zwischen gestuften und nicht gestuften Dimensionen von Kompetenz dar. Als gestuft werden Dimensionen bezeichnet, deren Ausprägungen eine hierarchische Ordnung zugrunde liegt bzw. für die eine solche Ordnung angenommen wird. Stufungen können entweder genutzt werden, um zu beschreiben, dass die einzelnen Ausprägungen qualitativ unterschiedliche Kompetenzniveaus abbilden (gestufte Strukturmodelle), oder aber, um zusätzlich zu modellieren, dass diese Niveaus im Lernen nacheinander durchlaufen werden (Entwicklungsmodelle; Schecker und Parchmann 2006). Nicht gestufte Dimensionen sehen alle Ausprägungen als gleichwertig an. Im Folgenden werden zunächst solche Dimensionen beschrieben, deren Ausprägungen typischerweise keine Annahme einer Stufung zugrunde liegt und für die eine solche Stufung aus unserer Sicht auch nicht naheliegend ist (vgl. Tab. 2). Im Anschluss daran werden (potenziell) stufbare Dimensionen fachinhaltlicher Kompetenz betrachtet (vgl. Tab. 3). 
Tab. 2 Übersicht über Dimensionen der Modellierung fachinhaltlicher Kompetenz und zugehörige Bereiche - Teil I (üblicherweise nicht gestufte Dimensionen)

\begin{tabular}{|c|c|c|}
\hline \multicolumn{2}{|l|}{ Dimension } & $\begin{array}{l}\text { Beispiele für mögliche Bereiche } \\
\text { Themenbereiche (HarmoS, EDK 2011) }\end{array}$ \\
\hline (1) Inhalt & $\begin{array}{l}\text { (1a) } \\
\text { Überfachlich }\end{array}$ & $\begin{array}{l}\text { Themenbereiche (HarmoS, EDK 2011) } \\
\text { Planet Erde } \\
\text { Bewegungen, Kraft, Energie } \\
\text { Wahrnehmung und Steuerung } \\
\text { Stoffe und Stoffveränderung } \\
\text { Lebewesen } \\
\text { Lebensräume und Lebensgemeinschaften } \\
\text { Mensch und Gesundheit } \\
\text { Natur, Gesellschaft, Technik - Perspektive } \\
\text { Crosscutting Concepts (NGSS, NGSS Lead States 2013) } \\
\text { Patterns } \\
\text { Cause and Effect } \\
\text { Scale, Proportion, and Quantity } \\
\text { Systems and System Models } \\
\text { Energy and Matter } \\
\text { Structure and Function } \\
\text { Stability and Change }\end{array}$ \\
\hline & $\begin{array}{l}\text { (1b) } \\
\text { Innerfachlich }\end{array}$ & $\begin{array}{l}\text { Basiskonzepte (Bildungsstandards Physik, KMK 2005c) } \\
\text { Energie } \\
\text { Materie } \\
\text { Wechselwirkung } \\
\text { System }\end{array}$ \\
\hline & & $\begin{array}{l}\text { Core Ideas (NGSS, z. B. Life sciences, NGSS Lead States 2013) } \\
\text { From Molecules to Organisms: Structures and Processes } \\
\text { Ecosystems: Interactions, Energy, and Dynamics } \\
\text { Heredity: Inheritance and Variation of Traits } \\
\text { Biological Evolution: Unity and Diversity }\end{array}$ \\
\hline & & $\begin{array}{l}\text { Stoffgebiete (TIMSS II, Baumert et al. 1998; TIMSS III, Baumert et al. 2000) } \\
\text { Geographie und Biowissenschaften } \\
\text { Biologie und Biowissenschaften } \\
\text { Physik } \\
\text { Technologie } \\
\text { Naturwissenschaftliches Arbeiten und Argumentieren }{ }^{\mathrm{a}} \rightarrow \text { Tab. } 1\end{array}$ \\
\hline & & $\begin{array}{l}\text { Sachgebiete (z. B. in TIMSS III für Physik, Baumert et al. 2000) } \\
\text { Mechanik } \\
\text { E-Lehre \& Magnetismus } \\
\text { Wärmelehre } \\
\text { Wellen und Schwingungen } \\
\text { Moderne Physik }\end{array}$ \\
\hline & & $\begin{array}{l}\text { Inhaltsbereich/Basiskonzept (Schecker und Parchmann 2006) } \\
\text { Energie } \\
\text { Materie } \\
\text { Nature of Science } \rightarrow \text { Tab. } 1 \\
\text { Thermodynamik } \\
\ldots\end{array}$ \\
\hline
\end{tabular}

\section{Dimensionierung fachinhaltlicher Kompetenz - Teil I: Dimensionen mit üblicherweise nicht gestuften Ausprägungen}

In den von uns analysierten Kompetenzmodellen konnten wir zwei verschiedene nicht gestufte Dimensionen identifizieren, die zur Beschreibung fachinhaltlicher Kompetenz genutzt werden: Die Dimension Inhalt (1) beschreibt die fachlichen Themengebiete, aus denen das Wissen stammt, das bei der Entfaltung der Kompetenz genutzt werden soll.
Die zweite Dimension, von uns als Kontext (2) bezeichnet, beschreibt die Situationsklassen, in denen das inhaltliche Wissen angewendet werden soll. Durch die Unterscheidung der beiden Dimensionen ist möglich, abzubilden, dass Schüler*innen z. B. die innerfachliche Anwendung eines bestimmten Wissenselementes (z.B. zu Energie) noch gelingen kann, während ihnen Anwendung in einem gesellschaftlichen Zusammenhang nicht mehr gelingt (Dimension (2a) in Tab. 2). Beide Dimensionen sind auch aus theoretischer Perspektive bedeutsam, da Kompetenz immer nur 
Tab. 2 (Fortsetzung)

\begin{tabular}{lll}
\hline Dimension & & Beispiele für mögliche Bereiche \\
\hline Kontext & (2a) & Anwendungsbereich (PISA 2015, OECD 2017) \\
& & Gesundheit und Krankheit \\
& Natürliche Ressourcen \\
& Umwelt bzw. Umweltqualität \\
& Gefahren \\
& Grenzen von Wissenschaft und Technologie \\
& Kontext (Schecker und Parchmann 2006) \\
& Innerfachlich \\
& Persönlich-gesellschaftliches Umfeld \\
& Professionelle Anwendung (Technik, Wissenschaft) \\
& Reichweite ${ }^{b}$ (PISA 2015, OECD 2017) \\
& (2b) Reich- & Persönlich (Selbst, Familie, Peers) \\
weite & Lokal/National \\
& Global
\end{tabular}

kursiv Benennung des Bereichs im jeweiligen Modell.

${ }^{a}$ Ausprägung des Bereichs passt nicht vollständig in die von uns konstruierte Dimension, der der Bereich zugeordnet wurde.

${ }^{\mathrm{b}}$ Bereich ist im Original nicht benannt, Benennung durch Autorengruppe

„bereichsspezifisch und auf einen begrenzten Sektor von Kontexten und Situationen bezogen" (Hartig und Klieme 2006, S. 129; s. a. Blömeke et al. 2015; Weinert 2001) beschrieben werden kann.

Bereits die Betrachtung der Dimension Inhalt (1) zeigt, dass, wie auch schon in der Beschreibung naturwissenschaftliche Kompetenz insgesamt, unterschiedliche $\mathrm{Zu}$ schnitte gewählt werden. Um diese grundsätzlichen Unterschiede bzgl. der Zuschnitte der einzelnen Dimensionen angemessen abbilden zu können, haben wir hier und auch im nächsten Abschnitt (vgl. Tab. 3) z.T. einzelne Dimensionen in mehrere Subdimensionen zerlegt. Beispielsweise werden in einigen Modellierungen für die inhaltliche Dimension sach- bzw. themenbezogene Zuschnitte verwendet, denen eine überfachliche Systematik zugrunde liegt (1a). Die Ausprägungen der entsprechenden Bereiche werden somit als für alle naturwissenschaftlichen Fächer bzw. Disziplinen gleichermaßen gültig angenommen und finden sich beispielsweise in Ländern, die innerhalb der Naturwissenschaften keine weitere Unterscheidung einzelner Disziplinen vornehmen (z. B. in den USA, siehe NRC 2012 oder der Schweiz, siehe D-EDK 2014). Andere Modellierungen lösen die Fächer bzw. Disziplinen auf oder schlagen für das Fach bzw. die Disziplin spezifische Sach- oder Themengebiete vor (1b). Für die Dimension Kontext (2) lassen sich die beiden von uns unterschiedenen Subdimensionen - Anwendung (2a) und Reichweite (2b) - unmittelbar aus der in PISA 2015 gewählten Modellierung ableiten, während andere Modelle nur eine der beiden Subdimensionen umfassen (z. B. (2a) in Schecker und Parchmann 2006).

Im Hinblick auf die Zerlegung einer Dimension in zwei potenziell voneinander unterscheidbare Subdimensionen ist anzumerken, dass diese Subdimensionen nicht voneinander unabhängig sein müssen, sondern sich Kompetenz aus der Verknüpfung ergibt. So wurden beispielsweise in PI-
SA 2015 die fünf verschiedenen Teilbereiche (bzw. Ausprägungen) der Anwendung (2a) mit den drei Teilbereichen des Bereichs Reichweite (2b) gekreuzt und so insgesamt $5 \times 3=15$ Kontexte generiert, die Kompetenz modellieren (OECD 2017). Auch kann z.B. zumindest jedes in den Bildungsstandards für das Fach Physik angeführte „Basiskonzept“ (1b) potenziell mit jedem im HarmoSModell unterschiedenen „Themenbereich“ (1a) kombiniert werden. Jedoch zeigt sich auch hier die Unterschiedlichkeit der einzelnen Modelle: Die in den NGSS genannten „Crosscutting Concepts“, die explizit nicht auf einzelne Fächer/Disziplinen bezogen sind, enthalten z.B. Teilbereiche (u. a. „Energy and Matter“; NRC 2012, S. 84), die wiederum in den Bildungsstandards für einen fach- bzw. disziplinspezifischen Zuschnitt genutzt werden (Energie als Teilbereich von Physik, Materie als ein Teilbereich von Chemie; KMK 2005b, 2005c). Zudem lässt sich erkennen, dass Bereiche aus einzelnen Modellen Teilbereiche in anderen Modellen bilden (können). Sowohl die „Basiskonzepte“ (Bildungsstandards) als auch die „Sachgebiete“ (TIMSS II) wären z.B. denkbare Teilbereiche des Bereichs Physik (,Stoffgebiet" bei TIMSS II).

\section{Dimensionierung fachinhaltlicher Kompetenz - Teil II: Dimensionen mit üblicherweise als gestuft bzw. stufbar angenommenen Ausprägungen}

Zur Beschreibung von Niveaus finden sich in verschiedenen Kompetenzmodellen vielfältige Ansätze, die wir fünf weiteren Dimensionen zugeordnet haben (Tab. 3 in fortgesetzter Nummerierung von Tab. 2). Eine erste stufbare Dimension beschreibt die zur Lösung einer Aufgabe erforderliche Vernetzung (3) von Wissenselementen. In Anlehnung an eine bereits von Commons et al. (1998) getroffenen Unterscheidung lassen sich zwei Subdimensionen (3a 
Tab. 3 Übersicht über Dimensionen der Modellierung fachinhaltlicher Kompetenz und zugehörige Bereiche - Teil II (gestufte bzw. als potenziell stufbar eingeschätzte Dimensionen)

\begin{tabular}{|c|c|c|c|}
\hline \multicolumn{2}{|c|}{ Dimension } & Beispiele für mögliche Bereiche & Erläuterungen \\
\hline \multirow{12}{*}{$\begin{array}{l}\text { (3) Ver- } \\
\text { netzung }\end{array}$} & \multirow{6}{*}{$\begin{array}{l}(3 a) \\
\text { Zahl zu } \\
\text { verbin- } \\
\text { dender } \\
\text { Elemen- } \\
\text { te }\end{array}$} & \multicolumn{2}{|c|}{ Komplexität (ESNaS; Kauertz et al. 2010) } \\
\hline & & Ein Fakt & - \\
\hline & & Zwei Fakten & $\begin{array}{l}\text { Der Übergang von Fakten zu Zusammenhängen ist eher der Subdimensi- } \\
\text { on (3b) zuzuweisen, da hier nicht die Anzahl, sondern der Charakter der } \\
\text { Verbindung verändert wird }\end{array}$ \\
\hline & & Ein Zusammenhang & - \\
\hline & & Zwei Zusammenhänge & - \\
\hline & & Übergeordnetes Konzept ${ }^{\mathrm{a}}$ & Eher Dimension (5b) zuzuweisen \\
\hline & \multirow{6}{*}{$\begin{array}{l}(3 b) \\
\text { Charak- } \\
\text { ter der } \\
\text { Verbin- } \\
\text { dung }\end{array}$} & \multicolumn{2}{|c|}{ Komplexität (MHC-C; Bernholt et al. 2009) } \\
\hline & & Unreflektiertes Erfahrungswissen $^{\mathrm{a}}$ & Eher Dimension (6) zuzuweisen \\
\hline & & Fakten $^{\mathrm{a}}$ & Eher Dimension (4) zuzuweisen \\
\hline & & Prozessbeschreibungen & - \\
\hline & & Lineare Kausalität & - \\
\hline & & Multivariate Interdependenz & - \\
\hline \multicolumn{2}{|l|}{ (4) } & \multicolumn{2}{|c|}{ Kognitive Anforderung (Schecker und Parchmann 2006) } \\
\hline \multirow[t]{9}{*}{$\begin{array}{l}\text { Denk- } \\
\text { prozess }\end{array}$} & & Divergentes Denken & $\begin{array}{l}\text { „Logisches Denken“: aus gegebenen Informationen die richtigen Schlüsse } \\
\text { ziehen }\end{array}$ \\
\hline & & Konvergentes Denken & $\begin{array}{l}\text { „Kreatives Denken“: vielfältige Antworten bzw. Lösungen für eine Aufgabe } \\
\text { bzw. ein Problem finden }\end{array}$ \\
\hline & & Umgang mit mentalen Modellen & - \\
\hline & & Umgang mit Zahlen & - \\
\hline & & Kognitive Aktivität (ESNaS; Kauert & .2010) \\
\hline & & Reproduzieren & Informationen erkennen und wiedergeben \\
\hline & & Selegieren & Informationen auswählen \\
\hline & & Organisieren & Informationen in einer Situation nutzen \\
\hline & & Integrieren & Informationen auf andere Situation übertragen \\
\hline \multirow{18}{*}{$\begin{array}{l}\text { (5) Ab- } \\
\text { strakti- } \\
\text { on }\end{array}$} & \multirow{13}{*}{$\begin{array}{l}(5 \mathrm{a}) \\
\text { Transfer }\end{array}$} & \multicolumn{2}{|c|}{ Anforderungsbereiche (Bildungsstandards; z. B. KMK 2005c) } \\
\hline & & Wiedergeben & - \\
\hline & & Anwenden & - \\
\hline & & Transfer & - \\
\hline & & \multicolumn{2}{|c|}{ Ausprägung (Schecker und Parchmann 2006) } \\
\hline & & Lebensweltlich $^{\mathrm{a}}$ & Eher Dimension (6) zuzuweisen \\
\hline & & Fachlich nominella/reproduktiv & Eher Dimension (6) zuzuweisen \\
\hline & & Aktiv anwenden & Reorganisation, naher Transfer (S. 58) \\
\hline & & Konzeptuell vertiefend & Ferner Transfer (S. 58) \\
\hline & & \multicolumn{2}{|c|}{ Kognitive Aktivität (Neumann et al. 2007) } \\
\hline & & Erinnern & Gelerntes wiedergeben (S. 112) \\
\hline & & Strukturieren & Bereits bekanntes Wissen neu ordnen (S. 112) \\
\hline & & Explorieren & Verbindung von neuem Wissen mit Gelerntem (S. 112) \\
\hline & $(5 b)$ & \multicolumn{2}{|c|}{ Konzeptualisierungsniveaus (von Aufschnaiter und Rogge 2010) } \\
\hline & \multirow[t]{4}{*}{$\begin{array}{l}\text { Genera- } \\
\text { lisierung }\end{array}$} & Explorativ & $\begin{array}{l}\text { Rein erkundendes Verhalten ohne [implizite] Aktivierung eines Konzeptes } \\
\text { möglich (S. 103) }\end{array}$ \\
\hline & & Intuitiv regelbasiert & $\begin{array}{l}\text { Zielsicheres Verhalten erforderlich, Konzept muss nicht verbalisiert werden } \\
\text { (S. 103) }\end{array}$ \\
\hline & & $\begin{array}{l}\text { Explizit regelbasiert, phänomenba- } \\
\text { siert }\end{array}$ & $\begin{array}{l}\text { Konzeptkenntnis muss explizit aktiviert werden, Konzept aus Erfahrung } \\
\text { ableitbar (S. 103) }\end{array}$ \\
\hline & & Explizit regelbasiert, modellbasiert & $\begin{array}{l}\text { Konzeptkenntnis muss explizit aktiviert werden, Konzept bezieht sich auf } \\
\text { nicht erfahrbare Sachverhalte (S. 103) }\end{array}$ \\
\hline
\end{tabular}


Tab. 3 (Fortsetzung)

\begin{tabular}{|c|c|c|}
\hline Dimension & Beispiele für mögliche Bereiche & Erläuterungen \\
\hline \multirow{5}{*}{$\begin{array}{l}\text { (6) An- } \\
\text { gemes- } \\
\text { senheit }\end{array}$} & \multicolumn{2}{|c|}{ Kompetenzniveaus (Kleickmann et al. 2010) } \\
\hline & Naive Vorstellungen & - \\
\hline & Zwischenvorstellungen & - \\
\hline & $\begin{array}{l}\text { Wissenschaftliche Vorstellungen, } \\
\text { wissenschaftliches Verständnis }\end{array}$ & Nennung richtiger Konzepte (S. 270) \\
\hline & $\begin{array}{l}\text { Wissenschaftliche Vorstellungen, } \\
\text { integriertes Verständnis }\end{array}$ & $\begin{array}{l}\text { Nennung richtiger Konzepte und explizite Ablehnung naiver Vorstellungen } \\
\text { (S. 270) }\end{array}$ \\
\hline \multirow{5}{*}{$\begin{array}{l}\text { (7) Ei- } \\
\text { genstän- } \\
\text { digkeit }\end{array}$} & \multicolumn{2}{|c|}{$\begin{array}{l}\text { Niveau (HarmoS; EDK 2011) } \\
\text { Merkmale, Funktionen, Objekte, Mechanismen benennen, unterscheiden und ... }\end{array}$} \\
\hline & $\ldots$ mit Alltags-/Sachbezug ordnen ${ }^{\mathrm{a}}$ & $\begin{array}{l}\text { Der Übergang von „Alltags-/Sachbezug“ zu „,naturwissenschaftlichem Be- } \\
\text { zug“ ist eher Dimension (1a) oder (2a) zuzuweisen }\end{array}$ \\
\hline & $\begin{array}{l}\text {... mit Alltags-/Sachbezug ordnen und } \\
\text { vergleichen }^{\mathrm{a}}\end{array}$ & $\begin{array}{l}\text { Der Übergang von „Ordnen“ zu „Ordnen und Vergleichen“ ist eher Dimen- } \\
\text { sion (4) zuzuweisen }\end{array}$ \\
\hline & $\begin{array}{l}\text {... mit vorgegebenem naturwissen- } \\
\text { schaftlichen Bezug ordnen und ver- } \\
\text { gleichen }\end{array}$ & - \\
\hline & $\begin{array}{l}\text {... mit eigenständigem naturwissen- } \\
\text { schaftlichen Bezug ordnen und ver- } \\
\text { gleichen }\end{array}$ & - \\
\hline
\end{tabular}

kursiv Benennung des Bereichs im jeweiligen Modell

${ }^{a}$ Ausprägung des Bereichs passt nicht vollständig in die von uns konstruierte Dimension, der der Bereich zugeordnet wurde. Die aus unserer Sicht am besten passende Dimension ist in der Spalte „Erläuterungen“ angegeben

${ }^{b}$ Erfordert in der Einschätzung der Kompetenz Kenntnis des vorlaufenden Unterrichts

und 3b) identifizieren: Vernetzung kann entweder im Sinne der Fähigkeit des Umgangs mit einer höheren Zahl zu verbindender Elemente verstanden werden $(3 \mathrm{a} ;$, ,nonhierarchical complexity“, S. 250) oder sich auf die Art bzw. den Charakter der Verbindung beziehen (3b; ,hierarchical complexity“, S. 250). Woitkowski und Riese (2017) weisen am Beispiel des ESNaS-Kompetenzmodells (Kauertz et al. 2010; vgl. Tab. 3) darauf hin, dass diese beiden Subdimensionen in Kompetenzmodellierungen z.T. zu einer Dimension aggregiert werden. Der Übergang von „einem Fakt" zu „zwei Fakten“ und von „einem Zusammenhang“ zu „Zwei Zusammenhängen“ bezieht sich z. B. auf die Anzahl der zu verbindenden Elemente, während sich der Übergang von „Fakten“ zu „Zusammenhängen“ eher auf den Charakter der Verbindung bezieht. Bei genauerer Betrachtung der von uns exemplarisch der Subdimension (3b) zugewiesenen Modellierung von Vernetzung im „Model of Hierarchical Complexity“ (MHC-C; Bernholt et al. 2009) zeigt sich zudem, dass eine weitere Ausschärfung erforderlich sein könnte: Die Subdimension (3b) kann sich auf die Art der Verbindung beziehen, z. B. unverbunden, monokausal oder interdependent. Sie kann aber auch auf einen zeitlichen Aspekt Bezug nehmen und darin eine Entwicklung von einer statischen Verbindung (Zusammenführung zeitlich invarianter Zustände, Ebenen der Objekte bis Ereignisse in von Aufschnaiter und Welzel 1997) zu einem dynamischen/funktionalen Zusammenhang modellieren (ab Ebene der Programme).
Die Dimension Denkprozesse (4) beschreibt, welche Art von gedanklichen Operationen bzw. „Denkvorgängen“ (Kauertz et al. 2010, S. 143) zur Bearbeitung einer Aufgabe ausgeführt werden müssen. Diese Dimension wurde bereits von Schecker und Parchmann (2006) identifiziert, dort allerdings als „kognitive Anforderung“ bezeichnet (S. 58). Unterschiedliche Denkprozesse, wie beispielsweise das konvergente und divergente Denken, implizieren zwar nicht automatisch eine Niveaustufung, in empirischen Untersuchungen zeigen sich jedoch Zusammenhänge zwischen den zur Bearbeitung einer Aufgabe erforderlichen Denkprozessen und der Lösungswahrscheinlichkeit (Rost et al. 2005). Es ist deshalb anzunehmen, dass diese Dimension potenziell stufbare Ausprägungen von Kompetenz umfasst. Tatsächlich werden verschiedene Denkprozesse bereits in manchen Modellierungen zur a priori Beschreibung von Stufen verwendet (z. B. in Kauertz et al. 2010; vgl. Tab. 3).

Die Abstraktion (5) lässt sich ebenfalls in substanziell verschiedene Modellierungen unterteilen. Zunächst kann sich Abstraktion darauf beziehen, ob und in welchem Umfang ein Transfer von bekannten Aufgaben und Problemen auf unbekannte Aufgaben und Problemen erforderlich ist (5a). Es ist dabei grundsätzlich plausibel anzunehmen, dass es sich hierbei um eine Stufung handelt: Einer bestimmten Person fällt es leichter, ein bestimmtes Wissenselement wiederzugeben, als es auf eine unbekannte Situation anzuwenden. Es ist jedoch zu bedenken, dass die Frage, ob eine Aufgabe Reproduktion oder Transfer erfordert, 
immer vom Vorwissen der Person abhängt, die die Aufgabe bearbeitet (z.B. Neumann et al. 2007). Der häufig gewählte Ansatz, das zur Bearbeitung einer Aufgabe erforderliche Wissen in den Aufgabenstamm zu integrieren (Ropohl et al. 2015), kann diesem Problem nur bedingt begegnen, da davon auszugehen ist, dass im alleinigen Lesen kaum angemessen erfasst werden kann, was noch grundsätzlich unbekannt oder unverstanden ist (z.B. Labudde et al. 2009; Vorholzer et al. 2016). Der Vergleich der Dimensionen (5a) und (4) zeigt exemplarisch auch, warum es problematisch ist, Aussagen über Gemeinsamkeiten (und Unterschiede) von Modellen alleine aus der Benennung von Dimensionen bzw. ihren Ausprägungen/Niveaus zu rekonstruieren: Oberflächlich lassen sich deutliche Parallelen zwischen (5a) und (4) erkennen, insbesondere für die beschriebenen Denkprozesse (z.B. ESNaS: reproduzieren, selegieren, etc. vgl. Tab. 3). Ein zentraler Unterschied ist jedoch, dass die in (4) beschriebenen Denkprozesse unabhängig von den Vorkenntnissen der Lernenden sind, während dies für die Einschätzung des geforderten Transfers nicht gilt. Beispielsweise kann ,konvergentes Denken“ oder das „Selegieren“ von Informationen (4) sowohl in bekannten (,Wiedergeben“/,Anwenden“) als auch in unbekannten Situationen (,Transfer“) erforderlich sein. Ein von Transfer unterscheidbarer zweiter Zugang zu Abstraktion bezieht sich auf den Grad der erforderlichen Generalisierung (5b). Ein Beispiel für eine Modellierung, die auf diese von uns rekonstruierte Subdimension Bezug nimmt, ist die in von Aufschnaiter und Rogge (2010) beschrieben Unterscheidung zwischen dem Umgang mit bestimmten Fällen (Ebenen der Exploration und des intuitiv regelbasierten Verhaltens) und der expliziten Angabe von Konzepten als Verallgemeinerungen (explizit regelbasiertes Verhalten). Hinweise auf eine Unterscheidung bzgl. dieser Subdimension zeigen sich beispielsweise auch im ESNaS-Modell (Kauertz et al. 2010), in dem u. a. zwischen „Fakten“ (eher geringe Generalisierung) und „übergeordnete Konzepte“ (eher hohe Generalisierung) unterschieden wird (Tab. 3, Subdimension (3b)).

Vielleicht etwas überraschend ist, dass wir kaum ein Modell identifizieren konnten, welches explizit eine Stufung der fachlichen Angemessenheit (6) vorsieht. Eine Ausnahme bildet hier das für den Grundschulbereich entwickelte Modell von Kleickmann et al., welches eine Stufung von „naiven Vorstellungen“ über „Zwischenvorstellungen“ bis zu „wissenschaftlichen Vorstellungen“ beschreibt (2010, S. 268). Viele weitere Modelle enthalten entsprechende Überlegungen implizit in Ausprägungen zu anderen Dimensionen. Es wird z.B. auf eher niedrigen Niveaus von lebensweltlichen oder alltagsorientierten Vorstellungen gesprochen, die sich zugunsten wissenschaftlicher Vorstellungen verändern (z. B. im Bereich „Komplexität“ (3b, Tab. 3) bei Bernholt et al. 2009, oder im Bereich „Aus- prägung“ (5a, Tab. 3) bei Schecker und Parchmann 2006). Einen möglichen Ansatzpunkt für die Graduierung von Kompetenz bzgl. der Dimension Angemessenheit stellen Learning Progressions dar (zsf. Alonzo 2012). Charakteristisch für Learning Progressions ist, dass fachliches Verständnis nicht dichotom (richtig oder falsch), sondern entlang mehrerer Zwischenstufen (,Levels“; Alonzo 2012, S. 96) beschrieben wird. Diese Zwischenstufen bilden z.T. graduelle Unterschiede bzgl. teilweise angemessener Verständnisse ab, welche sich auch für die Modellierung gestufter Ausprägungen eignen könnten. Im deutschen Sprachraum finden sich für den fachinhaltlichen Bereich z.B. Learning Progressions zu den Themen Energie (z.B. Neumann et al. 2013) und Materie (z.B. Hadenfeldt und Neumann 2012). Hier zeigt sich jedoch auch eine Herausforderung der Verknüpfung von Learning Progressions und den üblichen mehrdimensionalen Kompetenzmodellen: Eine Learning Progression ist i.d.R. auf einen Inhalt zugeschnitten (z.B. Energie, Materie), somit wäre für jede Ausprägung der Dimension Inhalt (2) ein spezifisches Set an Ausprägungen in mindestens einer weiteren Dimension erforderlich.

Als eine weitere Dimension konnte die Eigenständigkeit (7) aus dem Kompetenzmodell HarmoS Naturwissenschaften+ abgeleitet werden. Der Begriff ,abgeleitet“ ist hierbei bewusst gewählt, da das HarmoS-Modell, anders als die meisten Kompetenzmodelle, nicht global, sondern für jeden der insgesamt acht „Handlungsaspekte“ des Modells (u.a. „Fragen und untersuchen“ oder „Informationen erschließen“; z. B. Ramseier et al. 2011, S. 9; nicht in Tab. 2 und 3 enthalten) individuell verschiedene Niveaustufen beschreibt, und dabei auf sehr unterschiedliche Möglichkeit der Stufung zurückgreift (z. B. Adamina et al. 2010; Ramseier et al. 2011). Eine dieser Möglichkeiten, die sich aus unserer Sicht auch grundsätzlich zur Stufung von Kompetenz eignet, bezieht sich auf die Frage, inwiefern eine Aufgabe eigenständig bearbeitet werden muss. Das HarmoSModell unterscheidet hier beispielsweise die Komponenten ,angeleitet“, „teilweise eigenständig“ und „eigenständig“ (Adamina et al. 2010, S. 18; s. a. „Progressionslogiken“ im Schweizer Lehrplan 21, D-EDK 2014, S. 19). Hier zeigt sich, dass nicht alle Ausprägungen in einer Dimension sinnvoll mit allen Ausprägungen in einer anderen Dimension verbunden werden können. So ist beispielsweise für das „Reproduzieren“ (eine Ausprägung von Denkprozess (4)) eine Unterscheidung zwischen ,angeleitet“ und „eigenständig“ (mögliche Ausprägungen von Eigenständigkeit (7)) wenig zielführend. Für das „Organisieren“ oder „Integrieren“ bildet die Unterscheidung zwischen „,angeleitet“ und ,eigenständig“ jedoch relevante Ausprägungen von Kompetenz ab.

Die Tab. 2 und 3 zeigen in der Zusammenschau noch einmal deutlich, dass sich die in manchen Modellen be- 
schriebenen Ausprägungen nicht vollständig in eine von uns konstruierte Dimension einordnen lassen (in Tab. 2 und 3 mit einem hochgestellten ,a“ gekennzeichnet). Diese Unschärfe erklärt sich auf der einen Seite durch das Anliegen der Modelle, einen Auszug aus einem komplexen Kompetenzgefüge zu beschreiben (s. unten), auf der anderen Seite sind die verwendeten Begrifflichkeiten nicht immer hinreichend eindeutig. Auffällig ist beispielsweise, dass der Begriff der „Anforderung“ in den dokumentierten Modellen unterschiedlich genutzt wird, sodass wir Zuweisungen zu Denkprozess (4) und Abstraktion (5) vorgenommen haben. Der Begriff der "Anforderung“ ist zudem aus unserer Sicht zur Bezeichnung einer Dimension eines Modells nicht optimal gewählt, weil alle Dimensionen eines Modells zusammengenommen Anforderungen beschreiben, die durch Aufgaben abgebildet und erfasst werden.

\section{Erträge der vorgeschlagenen Systematik}

Kompetenzmodelle sind theoretisch oder empirisch abgeleitete Konstrukte, die für bestimmte Zwecke geschaffen werden. Sie bilden für den jeweiligen Zweck relevante Facetten von naturwissenschaftlicher Kompetenz ab, während andere Facetten u. a. zugunsten der Handhabbarkeit des Modells vernachlässigt werden. Damit weisen Kompetenzmodelle immer spezifische Grenzen und Einschränkungen auf, vor deren Hintergrund die aus ihnen abgeleiteten Aussagen diskutiert werden sollten. Im Sinne eines solchen Modellverständnisses ist uns wichtig zu betonen, dass es nicht Ziel des Beitrags ist, die Unschärfen von Dimensionen und Ausprägungen in bestehenden Kompetenzmodellen zu kritisieren, da diese für den Zweck der jeweiligen Modellierung durchaus zielführend und sinnvoll sein können. Aufgrund dieser (unvermeidbaren) Unschärfe und weil unsere Dimensionen in einem induktiven Verfahren generiert wurden, ist es durchaus möglich, dass andere Forscher*innen die Dimensionen anders benennen und/oder ggf. (etwas) andere Zuschnitte bzw. Zuweisungen vornehmen würden. Es ist auch zu bedenken, dass wir aufgrund unseres methodischen Vorgehens nur solche Dimensionen identifizieren konnten, die bereits in der Literatur beschrieben sind. Damit einher geht, dass eine möglicherweise erforderliche Ausdifferenzierung, wie sie sich z.B. innerhalb der Dimension (3b) andeutet, nur dann vorgenommen wurde, wenn entsprechende Subdimension in einem existierenden Modell identifiziert werden konnten. Trotz dieser Einschränkungen lässt sich anhand der Systematik zeigen, dass in den gegenwärtig vorliegenden Modellen zur Beschreibung fachinhaltlicher Kompetenz mindestens auf sieben (Haupt-)Dimensionen Bezug genommen wird (vgl. die zusammenfassende Darstellung in Tab. 4).
Die vorgestellte Systematik kann einen wichtigen Beitrag dazu leisten, einen strukturierten Überblick über das Forschungsfeld insgesamt zu erhalten, und eine Diskussionsgrundlage für begriffsbezogene Klärungsprozesse bieten. Vor diesem Hintergrund eignet sich die Systematik auch, um zwei der von Schecker und Parchmann formulierten Aufgaben für ,die fachdidaktische Forschung von und mit Kompetenzmodellen“ (2006, S. 63) aufzugreifen, die sich auf die Dimensionierung von fach(inhalt)licher Kompetenz beziehen.

Zu diesen Aufgaben gehörte die „Erforschung der Frage, welche Rolle Inhaltsbereiche, Prozesse, kognitive Anforderungen und Kontexte für die Struktur naturwissenschaftlicher Kompetenz spielen“ (Schecker und Parchmann 2006, S. 63) und das damit verbundene Ziel, die Dimensionierung eines Kompetenzmodells empirisch abzusichern. Bei Betrachtung der zahlreichen Modelle fachinhaltlicher (und prozessbezogener) Kompetenz zeigt sich, dass die fachdidaktische Forschung in der letzten Dekade deutliche Fortschritte bei der Beschreibung und Modellierung der Struktur naturwissenschaftlicher Kompetenz sowie deren empirischer Überprüfung gemacht hat. Mit diesem Fortschritt geht einher, dass die Vielfalt der Dimensionen, die zur Beschreibung von Kompetenz genutzt werden, eher zu- als abgenommen hat. In diesem Zusammenhang stellt sich u. a. die Frage, welche der identifizierten Dimensionen (und deren Subdimensionen) für die Beschreibung von fachinhaltlicher Kompetenz (oder auch von z. B. prozessbezogenen Kompetenzen; vgl. Abschn. 6) relevant sind. Dabei ist auch zu klären, inwiefern diese Dimensionen wechselseitig empirisch trennbar sind, bzw. welche Dimensionen sich ggf. zusammenführen lassen. Diese Fragen sollten nicht allein aus psychometrischer, sondern auch aus theoretischer und normativer Perspektive betrachtet werden (Labudde et al. 2009). Die vorgestellte Systematik bildet aus unserer Sicht einen wichtigen Ausgangspunkt für entsprechende Untersuchungen und Diskurse.

Als eine weitere Aufgabe formulierten Schecker und Parchmann (2006), dass es erforderlich sei, vermehrt und auch auf langen Zeitskalen Längsschnittstudien durchzuführen, um so die bestehenden Kompetenzstrukturmodelle um empirisch fundierte Entwicklungsmodelle zu ergänzen. Mit Blick auf die von uns rekonstruierten Dimensionen kann festgehalten werden, dass diese von Schecker und Parchmann in 2006 formulierte Aufgabe auch mehr als 10 Jahre später noch aktuell ist. Trotz der systematischen Recherche konnten wir kaum Modelle finden, die Entwicklung im (individuellen) Lernprozess in den Blick nehmen und entsprechend empirisch geprüft werden (s.a. Diskussion in Renkl 2012). Ansatzpunkte sehen wir z.B. in Arbeiten zu Learning Progressions (z.B. Alonzo und Steedle 2009; Hadenfeldt und Neumann 2012; Neumann et al. 2013) oder in Modellen, die sich mit der Entwicklung 
Tab. 4 Überblick über rekonstruierte Dimensionen fachinhaltlicher Kompetenz

\begin{tabular}{|c|c|}
\hline Hauptdimension & Beschreibung \\
\hline (1) Inhalt & $\begin{array}{l}\text { (1a) Überfachlich: fachunabhängiger Inhaltsbereich, aus dem die Kenntnisse stammen, die genutzt werden sollen (z. B.: Pla- } \\
\text { net Erde, Lebewesen, Mensch und Gesundheit) } \\
\text { (1b) Innerfachlich: fachspezifischer Inhaltsbereich, aus dem die Kenntnisse stammen, die genutzt werden sollen (z. B.: Me- } \\
\text { chanik, E-Lehre, Wärmelehre) }\end{array}$ \\
\hline (2) Kontext & $\begin{array}{l}\text { (2a) Anwendungsbereich: Lebensbereich, auf den die Kompetenz bezogen werden soll (z. B. Gesundheit, Umwelt) } \\
\text { (2b) Reichweite: Persönlicher Bezug zu dem Bereich, auf den die Kompetenz bezogen werden soll (z. B. lokal, national, glo- } \\
\text { bal) }\end{array}$ \\
\hline (3) Vernetzung & $\begin{array}{l}\text { (3a) Zahl der Verbindungen: Anzahl der Elemente gleicher Art, die miteinander verknüpft werden sollen (z. B. ein Fakt, zwei } \\
\text { Fakten, drei Fakten etc.). } \\
\text { (3b) Charakter der Verbindungen: Art des Zusammenhangs zwischen den Elementen, die miteinander verbunden werden } \\
\text { sollen (z. B. unverbunden, monokausal, interdependent) }\end{array}$ \\
\hline $\begin{array}{l}\text { (4) Denkpro- } \\
\text { zess }\end{array}$ & Art der kognitiven Operation, die ausgeführt werden soll (z. B. konvergentes Denken, divergentes Denken etc.) \\
\hline $\begin{array}{l}\text { (5) Abstrakti- } \\
\text { on }\end{array}$ & $\begin{array}{l}\text { (5a) Transfer: Bekanntheit der Situation, der Aufgaben oder des Problems, in dem die Kompetenz entfaltet werden soll (z. B. } \\
\text { vollständig bekannt/Reproduktion, ähnlich zu Bekanntem/Anwendung, vollständig unbekannt/Transfer) } \\
\text { (5b) Generalisierung: Grad der Verallgemeinerung, der bei der Entfaltung der Kompetenz erreicht werden soll (z. B. Betrach- } \\
\text { tung bestimmter Fälle, Explizierung von verallgemeinernden Konzepten) }\end{array}$ \\
\hline $\begin{array}{l}\text { (6) Angemes- } \\
\text { senheit }\end{array}$ & $\begin{array}{l}\text { Anspruch an die fachliche Richtigkeit bzw. fachliche Passung der eingesetzten Kompetenz/Fähigkeiten/Kenntnisse (z. B. } \\
\text { naives Verständnis, teilweise wissenschaftliches Verständnis, wissenschaftliches Verständnis) }\end{array}$ \\
\hline $\begin{array}{l}\text { (7) Eigenstän- } \\
\text { digkeit }\end{array}$ & $\begin{array}{l}\text { Umfang, in dem die Entfaltung der Kompetenz selbstständig erfolgen soll (z. B. angeleitet, teilweise eigenständig, eigenstän- } \\
\text { dig) }\end{array}$ \\
\hline
\end{tabular}

des Konzeptverständnisses befassen (u.a. von Aufschnaiter und Rogge 2010). Erstere basieren aktuell mehrheitlich auf Quasi-Längsschnitten und lassen damit nur begrenzt Rückschlüsse auf die auf ,intramentaler Ebene“ (Renkl 2012, S. 50) ablaufende Entwicklung zu, nehmen jedoch in der Modellierung bereits erkennbar individuelle Lernprozesse in den Blick. Das durch von Aufschnaiter und Rogge (2010) beschriebene Modell wird empirisch durch Längsschnitte gestützt, diese umfassen jedoch ein vergleichsweise kleines Zeitfenster von wenigen Schulstunden. Ein besonders vielversprechender Ausgangspunkt für die Modellierung von Kompetenzentwicklung scheint uns deshalb das Projekt „Development of Learning in Science“ (u. a. Bernholt et al. 2016) zu sein, das Längsschnittuntersuchungen zur Entwicklung des Konzeptverständnisses in Chemie über mehrere Klassenstufen hinweg umfasst.

Jenseits dieser grundsätzlichen Aufgaben für das Forschungsfeld lassen sich vor dem Hintergrund der Systematik auch eher spezifisch-methodische Herausforderungen für die Modellierung und Messung von Kompetenz diskutieren. Zentrale Herausforderungen sind aus unserer Sicht (A) die systematische Kontrolle von Ausprägungen und (B) die Präzision von Kompetenzbeschreibungen.

\section{(A) Systematische Kontrolle von möglichen Ausprägungen in einzelnen Dimensionen}

Die sieben rekonstruierten Dimensionen (Tab. 4) zeigen, dass in den Modellen fachinhaltlicher Kompetenz sehr unterschiedliche Facetten beschrieben werden. Die Varianz bezieht sich dabei nicht nur auf die in verschiedenen Mo- dellen genutzten Dimensionen, sondern auch darauf, wie diese Dimensionen in den einzelnen Modellen operationalisiert sind (z.B. bzgl. ihres Zuschnitts oder bzgl. der Ausprägungen, die sie umfassen). Es ist davon auszugehen, dass sich die identifizierte Varianz nicht allein dadurch begründen oder wesentlich verringern lässt, dass einzelne Bereiche und/oder Ausprägungen eines Modells in Bereiche/Ausprägungen anderer Modelle überführt werden können. Für fachdidaktische Forschung stellt sich deshalb die Frage des Umgangs mit dieser Varianz. Es ist ohne Zweifel wenig zielführend, bei der Untersuchung fachinhaltlicher Kompetenz alle Dimensionen gleichzeitig erfassen zu wollen. Ein solches Anliegen wäre kaum handhabbar und die zugehörige Modellierung so komplex, dass eine empirische Prüfung nicht mehr möglich erscheint (vgl. Schecker und Parchmann 2006). Vor diesem Hintergrund ist auch nachvollziehbar, dass in den vorliegenden Modellen z.T. in einer Dimension Ausprägungen ,zusammengezogen“ werden, die wir unterschiedlichen Dimensionen zugewiesen haben (vgl. Diskussion der Subdimensionen von Vernetzung (3) oben). Gleichzeitig ist die Betrachtung der Vielzahl von Dimensionen sowohl für die Entwicklung von Kompetenzmodellen als auch für Aussagen über Kompetenzen von Lernenden in mehrfacher Hinsicht hilfreich. Sie kann z.B. in der Entwicklung und Prüfung von a priori Modellen genutzt werden, um anzugeben, (a) welche Dimensionen (im Sinne der ersten Spalte in Tab. 2 bzw. 3) modelliert wurden, (b) welche Dimensionen nicht modelliert, aber mindestens bei der Konstruktion entsprechender Aufgaben kontrolliert wurden, und (c) welche Dimensionen weder bei der Modellierung noch bei der Konstruktion 
Tab. 5 Kompetenzstufen in TIMSS/III und exemplarische Zuweisung der Dimensionen aus Tab. 2 und 3

Stufe im Modell (entnommen aus Baumert et al. 2000; S. 44 ff., Kursivsetzung im Original)

Stufe 5: Überwinden von Fehlvorstellungen

Die fachdidaktische Forschung hat nachgewiesen, dass alltagsgebundene Fehlvorstellungen auch bei fortgeschrittenen Lernern oft anzutreffen sind. [...] Interessanterweise kennzeichnet die Überwindung solcher Fehlvorstellungen das höchste Kompetenzniveau der voruniversitären Physik

Stufe 4: Selbstständiges fachliches Argumentieren und Problemlösen

Auf dieser Kompetenzstufe werden auch offene Fragestellungen zur Oberstufenphysik, die eigenständige Lösungsansätze und zum Teil divergente Denkprozesse erfordern, mit hinreichender Sicherheit gelöst. [...]

Stufe 3: Anwendung physikalischer Gesetze zur Erklärung experimenteller Effekte auf Oberstufenniveau

Mit dieser Kompetenzstufe wird ein Kern physikalischer Kompetenz erreicht, nämlich die Kenntnis physikalischer Gesetze und klassischer Experimente, auf die jene Gesetze zutreffen. [...]

Stufe 2: Anwendung von Faktenwissen zur Erklärung einfacher Phänomene der Oberstufenphysik [...] Aufgaben, die für diese zweite Kompetenzstufe charakteristisch sind, beinhalten Themen [...] des Oberstufenunterrichts. Zur Lösung sind allerdings nur relativ einfache Faktenkenntnisse erforderlich $[\ldots]$

Stufe 1: Lösen von Routineaufgaben mit Mittelstufenwissen

Das unterste Kompetenzniveau indiziert einen erfolgreichen Umgang mit Standardstoffen der Mittelstufe
Dimension(en), auf die Bezug genommen wird (vgl. Tab. 2 und 3)

(6) Angemessenheit (fachlich unangemessene Vorstellungen bzw. deren Überwindung)

(1b) Inhalt (Physik: Oberstufe)

(4) Denkprozess (divergentes Denken)

(7) Eigenständigkeit (selbstständiges Problemlösen)

(1b) Inhalt (Physik: Gesetze und experimentelle Effekte der Oberstufe)

(1b) Inhalt (Physik: Phänomene der Oberstufe)

(5a) Transfer (Anwendung von Faktenwissen)

(1b) Inhalt (Physik: Standardstoff der Mittelstufe) berücksichtigt wurden. Die systematische Aufschlüsselung von modellierten Dimensionen und Ausprägungen bzw. der transparente Umgang mit dem Fehlen einer (vollständigen) Kontrolle selbiger kann u. a. dort relevant werden, wo theoretisch als trennbare angenommene Dimensionen bei einer empirischen Prüfung unerwartet zusammenfallen oder mehrere Studien zur Dimensionalität der gleichen Kompetenz zu unterschiedlichen Befunden gelangen. In dieser Funktion ist die Systematik sowohl auf Kompetenz im Sinne von Dispositionen als auch auf Kompetenz im Sinne von gezeigter Performanz anwendbar: Sollen z. B. valide Rückschlüsse auf das Vorhandensein bzw. die Ausprägung von Dispositionen gezogen werden, bedarf es Aufgaben, die sich möglichst nur im Hinblick auf die für diese Dispositionen als relevant angenommenen Merkmale unterscheiden. In gleicher Weise bedarf es für eine valide Schätzung der Güte von gezeigter Performanz möglichst kontrollierter und vergleichbarer Situationen, in denen diese Performanz beobachtet werden soll. ${ }^{3}$

Der Versuch, eine Systematik für Dimensionen von Kompetenz zu beschreiben, ist nicht nur dort ertragreich, wo Kompetenz modelliert bzw. a priori entwickelte Kompe-

\footnotetext{
3 Variablenkontrolle ist hierbei nicht nur im Hinblick auf die Dimensionen fachinhaltlicher Kompetenz relevant, sondern betrifft auch nicht-fachspezifische Kompetenzen. Beispielsweise sollte bei der Aufgabenkonstruktion darauf geachtet werden, dass die Anforderungen an die Lese- oder die Rechtschreibkompetenz der Lernenden nicht unbeabsichtigt über die Aufgaben hinweg variiert werden. Zur Kontrolle solcher Merkmale kann grundsätzlich eine vergleichbare Systematik erstellt und angewendet werden (z.B. zu den Dimensionen/Ausprägungen von Lesekompetenz). Da es sich hierbei jedoch nicht um eine fachspezifische Kompetenz handelt, haben wir keine entsprechenden Dimensionen in Tab. 2 und 3 aufgenommen.
}

tenzmodelle mit variablenkontrolliert generierten Aufgaben geprüft werden sollen. Eine solche Systematik kann auch für die Konstruktion sogenannter post-hoc Modelle (,deskriptive Modelle“ nach Schecker und Parchmann 2006) genutzt werden, bei deren Entwicklung aus Gemeinsamkeiten von Aufgaben mit ähnlicher Lösungswahrscheinlichkeit Merkmale unterschiedlicher Kompetenzstufen abgeleitet werden. Dieses Vorgehen kommt beispielsweise dann zum Einsatz, wenn keine gesicherten oder theoretisch fundierten Annahmen über mögliche Kompetenzstufen vorliegen (z.B. Mayer und Wellnitz 2014). Das Fehlen solcher Annahmen erschwert die systematische Kontrolle der zur Beschreibung unterschiedlicher Kompetenzanforderungen relevanten Dimensionen (und Ausprägungen), weshalb post-hoc generierte Modelle zwangsläufig Dimensionen nicht immer optimal trennen. Dies zeigt sich z.B. in der Einordnung der Modellierung der Kompetenzstufen bei TIMSS/III (Baumert et al. 2000) in unsere Systematik (vgl. Tab. 5). So werden z.B. die Stufen 1 und 3 ausschließlich über die Dimension Inhalt (1) beschrieben, während die Beschreibungen der Stufen 2 und 4 zusätzlich die Dimensionen Denkprozesse (4) oder Transfer (5a) aufgreifen. In Stufe 5 wird der Inhalt nicht mehr beschrieben, sondern nur noch die Angemessenheit (6). Interessant ist zudem, dass laut Baumert et al. die Kompetenzstufe 2 die Lösung von Aufgaben ermöglicht, welche „relativ einfache Faktenkenntnisse" (2000, S. 44) erfordern. Welcher von unseren Dimensionen dieses „einfach“ zuzuordnen ist, konnten wir nicht aus der Beschreibung rekonstruieren. Es wäre denkbar, dass mit „einfach“ etwas gemeint ist, was in unsere Systematik bereits in einer anderen Dimension beschrieben wird (z. B. Inhalte, die wenig Vernetzung (3) erfordern). Es 
ist es aber auch möglich, dass auf eine weitere Dimension Bezug genommen wird, die in anderen Modellen fehlt und deshalb nicht in unserer Systematik enthalten ist. Das Fehlen einer präzisen Beschreibung dazu, was mit „einfach“ gemeint ist bzw. was einen einfachen von einem schweren Inhalt unterscheidet, erschwert somit den Vergleich bzw. die gezielte Einordnung des Merkmals dieser Kompetenzstufe. In ähnlicher Weise wird nicht klar, welche Denkprozesse und welches $\mathrm{Ma}$ an Eigenständigkeit eigentlich in den Aufgaben zu den Kompetenzstufen 1, 2, 3, 5 und 6 erforderlich war. Damit lässt sich nicht gut nachvollziehen, was genau Stufe 4 anspruchsvoller macht als Stufe 3: Die Variation des Inhalts, der Denkprozesse und/oder der Eigenständigkeit? Für eine vergleichende Betrachtung der in verschiedenen Gruppen (z. B. Klassen, Schulen, Länder ...) vorhandenen Kompetenzen ist ein solches Fehlen systematischer Kontrolle vermutlich eher unproblematisch; es lässt sich zwar nicht mehr genau sagen, was Schüler*innen in Gruppe A besser können als in Gruppe B, das Vorhandensein eines Kompetenzunterschieds lässt sich aber dennoch zeigen. Differenziertere Deutungen der Ergebnisse werden jedoch ohne eine kontrollierte und möglichst präzise Beschreibung von Dimensionen sowie deren Ausprägungen erheblich erschwert. Soll ein Modell z.B. dazu genutzt werden, Hinweise für die Förderung von Schüler*innen abzuleiten, ist eine systematische Kontrolle unverzichtbar, damit die für die jeweiligen Niveaustufen relevanten Lernbedarfe spezifiziert werden können.

\section{(B) Präzisierung von Kompetenzbeschreibungen}

Kompetenzmodelle werden typischerweise in einem mehrdimensionalen Koordinatensystem dargestellt, dessen Achsen die einzelnen Dimensionen der modellierten Kompetenz repräsentieren (siehe z. B. Übersicht in Bernholt et al. 2009; Schecker und Parchmann 2006). Um von dieser abstrakten Darstellung zu Aufgaben für eine Kompetenzmessung zu gelangen, bedarf es konkreter Kompetenzbeschreibungen. Hierbei ist eine Vernetzung der im Zuge der Modellierung im Idealfall streng getrennten Dimensionen unvermeidbar. So lässt sich beispielsweise im ESNaS-Modell (Kauertz et al. 2010) mit dem Bereich „Komplexität“ (z.B. Fakt, Fakten, Zusammenhang etc.) alleine noch keine Kompetenz beschreiben. Erst durch Hinzuziehen der ,,kognitiven Prozesse“ (z. B. reproduzieren, selegieren, etc.) und des „Kompetenzbereichs“ (z.B. Nutzung fachlicher Konzepte, Erkenntnisgewinnung, Kommunikation, Bewertung) wird deutlich, was mit den Fakten oder Zusammenhängen gekonnt werden soll und aus welcher inhaltlichen Domäne die Fakten bzw. Zusammenhänge stammen sollen. Eine mögliche Kompetenzbeschreibung zum ESNaS-Modell könnte z. B. lauten:
Die Schüler*innen reproduzieren einen Zusammenhang zum naturwissenschaftlichen Fachwissen.

Anhand dieser exemplarischen Kompetenzbeschreibung wird zunächst einmal deutlich, dass Kompetenzmodelle nicht „unterkomplex“ sein können. Es werden mindestens zwei Achsen (im Sinne von Dimensionen) zur Festlegung des Operators (hier als kognitiver Prozess) und des Gegenstandes (hier als Komplexität) sowie ein „Fixpunkt“ (im Sinne einer Ausprägung in einer dritten Dimension) zur Festlegung eines spezifischen Inhalts benötigt (z. B. Kauertz et al. 2010). Eine Beschreibung, die nur zwei Dimensionen umfasst, ist entweder unpräzise - z.B., weil offenbleibt, aus welchem Inhaltsbereich Fakten reproduziert werden sollen (Die Schüler*innen reproduzieren Fakten) - oder sinnlos (Die Schüler*innen reproduzieren Energie). Das oben beschriebene Problem der Vermischung von Dimensionen in einem eindimensionalen Kompetenzmodell (vgl. Tab. 5) könnte demzufolge auch damit zusammenhängen, dass solche Modelle grundsätzlich entweder nicht vollständig variablenkontrolliert angelegt sein können, oder aber $\mathrm{zu}$ vergleichsweise trivialen Kompetenzausprägungen in dieser einen Dimension führen:

Ausprägung 1: Die Schüler*innen reproduzieren einen Fakt zu Energie.

Ausprägung 2: Die Schüler*innen selegieren einen

Fakt zu Energie.

Ausprägung 3: ...

Aus dieser exemplarischen Betrachtung wird deutlich, dass Kompetenzbeschreibungen erforderlich sind, um eine Brücke zwischen einem zwangsläufig eher abstrakten Kompetenzmodell und den konkreten Aufgaben zur Erfassung der im Modell beschriebenen Kompetenz herzustellen. Ohne solche Beschreibungen ist nur schwer nachvollziehbar, ob auf der Modellierungsebene ähnlich aussehende Modelle bei ihrer Überprüfung auch in ähnliche Aufgaben „übersetzt“" werden und inwiefern damit z. B. die Vergleichbarkeit der Ergebnisse gegeben ist. Kompetenzbeschreibungen würden zudem dabei helfen, besser nachvollziehen $\mathrm{zu}$ können, welche Variablen bzw. Dimensionen bei der Übersetzung des Kompetenzmodells in Aufgaben (nicht) in den Blick genommen wurden. Es wäre deshalb aus unserer Sicht zielführend, wenn in Forschungsbeiträgen neben den Beschreibungen der Dimensionen eines Kompetenzmodells verstärkt auch konkrete Beschreibungen der als zugehörig angenommen Kompetenzen angegeben würden.

\section{Transferpotenzial der Systematisierung}

Neben der Beschreibung fachinhaltlicher Kompetenzen wurde in den vergangenen Jahren verstärkt auch die Model- 
lierung prozessbezogener Kompetenzen von Schüler*innen in den Blick genommen (vgl. Tab. 1). Auch hier liegen bereits einige Modelle vor (im Bereich Erkenntnisgewinnung z.B. bei Kohlhauf et al. 2011; Nehring 2014; Upmeier zu Belzen und Krüger 2010; Wellnitz et al. 2012; Wellnitz und Mayer 2013; im Bereich Kommunikation z.B. bei Kulgemeyer und Schecker 2009; Ziepprecht et al. 2017; im Bereich Bewerten z.B. bei Hostenbach et al. 2011), die jedoch in ihrer Beschreibung der jeweiligen Kompetenzen nur wenige gestufte Dimensionen vorsehen. Aus unserer Sicht stellt sich deshalb u.a. die Frage, inwiefern sich die zur Stufung fachinhaltlicher Kompetenz genutzten Dimensionen (vgl. Tab. 3) auch auf prozessbezogenen Kompetenzen anwenden lassen. Erste Überlegungen dazu finden sich u.a. im Modell von Schecker und Parchmann (2006) und im ESNaS-Modell (z.B. Kauertz et al. 2010), die beide jeweils eine Dimension „Kompetenzbereich“ enthalten (vgl. Tab. 1) und damit annehmen, dass sich alle anderen beschriebenen Dimensionen gleichermaßen auf inhaltliche und auf prozessbezogene Kompetenzen anwenden lassen. Im Zusammenhang mit dem ESNaSModell gibt es zudem bereits erste empirische Evidenz, die diese Annahme für die Dimensionen Vernetzung - Zahl $z u$ verbindender Elemente (3a) und Denkprozess (4) für die Kompetenzbereiche Fachwissen und Erkenntnisgewinnung stützt (Kauertz et al. 2010; Wellnitz et al. 2012). Offen bleibt jedoch u.a., ob sich auch die anderen fünf (Sub-)Dimensionen (3b, 5a, 5b, 6, 7; Tab. 3) auf den Bereich Erkenntnisgewinnung anwenden lassen und inwiefern die gestuften Dimensionen grundsätzlich zur Modellierung anderer prozessbezogener Kompetenzen (z.B. Kommunikation und Bewertung) geeignet sind.

Gleichzeitig sollte der Frage nachgegangen werden, welche weiteren, für fachinhaltliche Kompetenzen irrelevanten oder zumindest nicht beschriebenen, Dimensionen für die Modellierung prozessbezogener Kompetenzen genutzt werden können. Dazu könnte das von uns in Abschn. 3 skizzierte Verfahren sowie unsere Art der Aufbereitung der Ergebnisse (Abschn. 4) auf Modelle zu anderen Kompetenzbereichen, wie z. B. Erkenntnisgewinnung, Kommunikation oder Bewertung, angewendet werden, um so die jeweils zur Beschreibung verwendeten Dimensionen zu identifizieren. Das Ergebnis der Entwicklung einer analogen Systematik (bzw. mehrerer Systematiken) für prozessbezogene Kompetenzen könnte einerseits auf Modellierungen fachinhaltlicher Kompetenz rückbezogen werden, um beispielsweise zu prüfen, inwiefern die identifizierten Dimensionen auch dort relevanten sind. Andererseits wäre ein solcher Systematisierungsversuch vermutlich auch deshalb hilfreich, weil u.a. das Problem der Kontrolle relevanter Variablen (Dimensionen und Ausprägungen) nicht nur Modelle fachinhaltlicher Kompetenz zu betreffen scheint. Dies zeigt sich z.B. im Bereich Erkenntnisgewinnung im Diskurs um den
Zuschnitt experimentbezogener Kompetenz (zsf. Vorholzer et al. 2016; s. a. Wellnitz und Mayer 2013): Hier wird in einer Reihe unterschiedlicher Arbeiten diskutiert, ob es sich bei experimentbezogener Kompetenz um eine Kompetenz handelt oder ob diese Kompetenz in mehrere empirisch trennbare Teilkompetenzen zerlegt werden kann. Typischerweise werden mindestens drei Teilkompetenzen angenommen, die sich in ihrem Zuschnitt an einem idealisierten Modell der Schritte naturwissenschaftlicher Erkenntnisgewinnung orientieren (z.B. Fragen und Hypothesen formulieren, Untersuchungen planen und durchführen, Daten auswerten und interpretieren; siehe z.B. Übersichten in Vorholzer et al. 2016; Emden und Sumfleth 2016). Empirische Untersuchungen dazu, ob mehrdimensionale Modelle, welche die jeweils unterschiedenen Teilkompetenzen auflösen, tatsächlich besser zu den Daten passen als eindimensionale Modelle, kommen zu unterschiedlichen Ergebnissen. Wir vermuten, dass eine Ursache für die unterschiedlichen Befunde die Varianz in den nicht modellierten bzw. kontrollierten Dimensionen experimentbezogener Kompetenz liegt (ausführlich in Vorholzer et al. 2016). Dass auch in Modellen aus dem Bereich Erkenntnisgewinnung Kompetenzbeschreibungen nicht immer hinreichend präzise sind und z. T. mehrere Dimensionen „entlang einer Achse“ variiert werden, zeigt sich z. B. im Kompetenzstufenmodell zu den Bildungsstandards im Fach Chemie (KMK 2013). Dort werden beim Übergang von Kompetenzstufe III (,Anwenden von naturwissenschaftlichen Methoden der Erkenntnisgewinnung und Modellen in einfachen fachlichen Zusammenhängen“) zu Kompetenzstufe IV (,Begründetes Auswählen und Nutzen von naturwissenschaftlichen Methoden [...] in komplexen Zusammenhängen“; KMK 2013, S. 46, eigene Hervorhebung) sowohl der Denkprozess (4) als auch die „Komplexität“ (ohne nähere Beschreibung nicht eindeutig einer Dimension zuzuordnen) variiert.

Unser methodisches Vorgehen und die resultierende Systematik haben nicht nur für andere Kompetenzbereiche einen Mehrwert, sondern auch für die Übertragung auf andere Zielgruppen (z.B. fachinhaltliche Kompetenz von (angehenden) Lehrkräften). Dies zeigt sich z.B. auch bei der Betrachtung des von Woitkowski und Riese (2017) vorgeschlagenen Kompetenzniveaumodells des physikalischen Fachwissens von Studierenden, welches u.a. die Dimensionen (3b) Vernetzung - Charakter der Verbindung (dort als „hierarchische Komplexität“ bezeichnet; S. 41) und (1b) Inhalt - Innerfachlich (dort als „Inhaltsbereich“ bezeichnet; S. 41) aufgreift. Es wäre aus unserer Sicht deshalb einerseits sinnvoll zu untersuchen, inwiefern die vergleichsweise vielfältigen Dimensionen, die zur Modellierung der fachinhaltlichen Kompetenz von Schüler*innen genutzt werden, auch für andere Zielgruppen nutzbar sind. Im Zuge einer solchen Erweiterung bieten sich möglicherweise auch Längsschnittstudien an, um stärker als 
bisher die Entwicklung auf ,intramentaler Ebene“ (vgl. Renkl 2012, S. 50) in den Blick zu nehmen, dabei bisher beschriebene Niveaus im Hinblick auf ihre zeitliche Abfolge weiter auszudifferenzieren und perspektivische Hinweise für Kompetenzentwicklungsmodelle abzuleiten. Andererseits kann geprüft werden, inwiefern das von uns beschriebene Vorgehen zur Identifikation der Dimensionen und Ausprägungen anderer Kompetenzen geeignet ist. Die in solchen Prozessen für unterschiedliche Zielgruppen und/oder Kompetenzbereiche identifizierte Dimensionen könnten anschießend mithilfe von Expertenbefragungen auf Überschneidungen geprüft werden, um zu explorieren, inwiefern sich hier Hinweise für kohärentere Kompetenzbeschreibungen ableiten lassen. Sollten sich dabei z. B. für verschiedene Kompetenzbereiche ähnliche Dimensionen identifizieren lassen, könnte deren wechselseitiger Vergleich perspektivisch zu einer Ausschärfung und/oder zu einer Vereinheitlichung der Beschreibung führen und so insgesamt zu einer höheren Kohärenz von Kompetenzbeschreibungen beitragen.

Funding Open Access funding provided by Projekt DEAL.

Open Access Dieser Artikel wird unter der Creative Commons Namensnennung 4.0 International Lizenz veröffentlicht, welche die Nutzung, Vervielfältigung, Bearbeitung, Verbreitung und Wiedergabe in jeglichem Medium und Format erlaubt, sofern Sie den/die ursprünglichen Autor(en) und die Quelle ordnungsgemäß nennen, einen Link zur Creative Commons Lizenz beifügen und angeben, ob Änderungen vorgenommen wurden.

Die in diesem Artikel enthaltenen Bilder und sonstiges Drittmaterial unterliegen ebenfalls der genannten Creative Commons Lizenz, sofern sich aus der Abbildungslegende nichts anderes ergibt. Sofern das betreffende Material nicht unter der genannten Creative Commons Lizenz steht und die betreffende Handlung nicht nach gesetzlichen Vorschriften erlaubt ist, ist für die oben aufgeführten Weiterverwendungen des Materials die Einwilligung des jeweiligen Rechteinhabers einzuholen.

Weitere Details zur Lizenz entnehmen Sie bitte der Lizenzinformation auf http://creativecommons.org/licenses/by/4.0/deed.de.

\section{Literatur}

Adamina, M., Labudde, P., Gingins, F., Nidegger, C., Bazzigher, L., Bringold, B., et al. (2010). Naturwissenschaften - Wissenschaftlicher Kurzbericht und Kompetenzmodell. https://edudoc.ch/static/ web/arbeiten/harmos/harmoS_kurzbericht_neu.pdf. Zugegriffen: 2. Aug. 2018.

Alonzo, A.C. (2012). Learning progressions: significant promise, significant challenge. Zeitschrift für Erziehungswissenschaft, 15(1), 95-109.

Alonzo, A.C., \& Steedle, J.T. (2009). Developing and assessing a force and motion learning progression. Science Education, 93(3), 389-421.

Aufschnaiter, C. v., \& Hofmann, J. (2014). Kompetenz und Wissen. Wechselseitige Zusammenhänge und Konsequenzen für die Unterrichtsplanung. Der mathematische und naturwissenschaftliche Unterricht, 67(1), 10-16.

Aufschnaiter, C. v., \& Rogge, C. (2010). Wie lassen sich Verläufe der Entwicklung von Kompetenz modellieren? Zeitschrift für Didaktik der Naturwissenschaften, 16, 95-114.
Aufschnaiter, S. v., \& Welzel, M. (1997). Wissensvermittlung durch Wissensentwicklung. Das Bremer Komplexitätsmodell zur quantitativen Beschreibung von Bedeutungsentwicklung und Lernen. Zeitschrift für Didaktik der Naturwissenschaften, 3(2), 43-57.

Baumert, J., Lehmann, R., Lehrke, M., Clausen, M., Hosenfeld, I., Neubrand, J., et al. (1998). Testaufgaben der Naturwissenschaften TIMSS 7./8. Klasse. Population, Bd. 2. Berlin: Max-PlanckInstitut für Bildungsforschung.

Baumert, J., Bos, W., Brockmann, J., Gruehn, S., Klieme, E., Köller, O., et al. (2000). TIMSS/III-Deutschland - Der Abschlussbericht. Zusammenfassung ausgewählter Ergebnisse der Dritten Internationalen Mathematik- und Naturwissenschaftsstudie zur mathematischen und naturwissenschaftlichen Bildung am Ende der Schullaufbahn. Berlin: Max-Planck-Institut für Bildungsforschung.

Bernholt, S., Köhler, C., \& Broman, K. (2016). Die Verständnisentwicklung zentraler Fachkonzepte im Chemieunterricht der Sekundarstufe. In C. Maurer (Hrsg.), Authentizität und Lernen - das Fach in der Fachdidaktik. Gesellschaft für Didaktik der Chemie und Physik, Jahrestagung in Berlin 2015. (S. 223-225). Kiel: IPN.

Bernholt, S., Parchmann, I., \& Commons, M.L. (2009). Kompetenzmodellierung zwischen Forschung und Unterrichtspraxis. Zeitschrift für Didaktik der Naturwissenschaften, 15, 219-245.

Blömeke, S., Gustafsson, J.-E., \& Shavelson, R. J. (2015). Beyond dichotomies. Zeitschrift für Psychologie, 223(1), 3-13.

Commons, M.L., Trudeau, E. J., Stein, S. A., Richards, F. A., \& Krause, S. R. (1998). Hierarchical complexity of tasks shows the existence of developmental stages. Developmental Review, 18(3), 237-278.

D-EDK (2014). Lehrplan 21. Natur, Mensch, Gesellschaft. Luzern: Deutschschweizer Erziehungsdirektoren-Konferenz.

EDK (2011). Grundkompetenzen für die Naturwissenschaften. Nationale Bildungsstandards. Bern: Schweizerische Konferenz der kantonalen Erziehungsdirektoren.

Emden, M., \& Sumfleth, E. (2016). Assessing students' experimentation processes in guided inquiry. International Journal of Science and Mathematics Education, 14(1), 29-54.

Hadenfeldt, J. C., \& Neumann, K. (2012). Die Erfassung des Verständnisses von Materie durch Ordered Multiple Choice Aufgaben. Zeitschrift für Didaktik der Naturwissenschaften, 18, 317-338.

Hartig, J., \& Klieme, E. (2006). Kompetenz und Kompetenzdiagnostik. In K. Schweizer (Hrsg.), Leistung und Leistungsdiagnostik (S. 127-143). Heidelberg: Springer.

Hostenbach, J., Fischer, H.E., Kauertz, A., Mayer, J., Sumfleth, E., \& Walpuski, M. (2011). Modellierung der Bewertungskompetenz in den Naturwissenschaften zur Evaluation der Nationalen Bildungsstandards. Zeitschrift für Didaktik der Naturwissenschaften, 17, 261-288.

Kauertz, A., Fischer, H.E., Mayer, J., Sumfleth, E., \& Walpuski, M. (2010). Standardbezogene Kompetenzmodellierung in den Naturwissenschaften der Sekundarstufe I. Zeitschrift für Didaktik der Naturwissenschaften, 16, 135-153.

Kleickmann, T., Hardy, I., Möller, K., Pollmeier, J., Tröbst, S., \& Beinbrech, C. (2010). Die Modellierung naturwissenschaftlicher Kompetenz im Grundschulalter. Theoretische Konzeption und Testkonstruktion. Zeitschrift für Didaktik der Naturwissenschaften, 16, 265-283.

Klieme, E., Avenarius, H., Blum, W., Döbrich, P., Gruber, H., Prenzel, M., et al. (2007). Zur Entwicklung nationaler Bildungsstandards. Eine Expertise. Berlin: Bundesministerium für Bildung und Forschung.

KMK (2013). Kompetenzstufenmodelle zu den Bildungsstandards im Fach Chemie für den Mittleren Schulabschluss. Kompetenzbereiche Fachwissen und Erkenntnisgewinnung. https://www.iqb. hu-berlin.de/bista/ksm/KSM_Chemie_1.pdf. Zugegriffen: 2. Juli 2019.

KMK (2005a). Bildungsstandards im Fach Biologie für den Mittleren Schulabschluss (Jahrgangsstufe 10). München: Luchterhand. 
KMK (2005b). Bildungsstandards im Fach Chemie für den Mittleren Schulabschluss (Jahrgangsstufe 10). München: Luchterhand.

KMK (2005c). Bildungsstandards im Fach Physik für den Mittleren Schulabschluss (Jahrgangsstufe 10). München: Luchterhand.

Koenen, J., Emden, M., \& Sumfleth, E. (2017). Naturwissenschaftlichexperimentelles Arbeiten. Zeitschrift für Didaktik der Naturwissenschaften, 23, 81-98.

Kohlhauf, L., Rutke, U., \& Neuhaus, B. (2011). Entwicklung eines Kompetenzmodells zum biologischen Beobachten ab dem Vorschulalter. Zeitschrift für Didaktik der Naturwissenschaften, 17, 203-222.

Kulgemeyer, C., \& Schecker, H. (2009). Kommunikationskompetenz in der Physik: Zur Entwicklung eines domänenspezifischen Kommunikationsbegriffs. Zeitschrift für Didaktik der Naturwissenschaften, 15, 131-153.

Kulgemeyer, C. \& Schecker, H. (2013). Schülerinnen und Schüler erklären Physik - Modellierung, Diagnostik und Förderung von Kommunikationskompetenz im Physikunterricht. In M. BeckerMrotzek, K. Schramm, E. Thürmann \& H. Vollmer (Hrsg.), Sprache im Fach. Sprachlichkeit und fachliches Lernen (S. 225-240). Münster: Waxmann.

Labudde, P., Duit, R., Fickermann, D., Fischer, H., Harms, U., Mikelskis, H., et al. (2009). Schwerpunkttagung „Kompetenzmodelle und Bildungsstandards: Aufgaben für die naturwissenschaftsdidaktische Forschung". Zeitschrift für Didaktik der Naturwissenschaften, 15, 343-370.

Mayer, J., \& Wellnitz, N. (2014). Die Entwicklung von Kompetenzstrukturmodellen. In D. Krüger, I. Parchmann \& H. Schecker (Hrsg.), Methoden in der naturwissenschaftsdidaktischen Forschung (S. 19-29). Heidelberg, Berlin, New York: Springer.

Mayring, P., \& Fenzl, T. (2014). Qualitative Inhaltsanalyse. In N. Baur \& J. Blasius (Hrsg.), Handbuch Methoden der empirischen Sozialforschung (S. 543-556). Wiesbaden: Springer.

Messick, S. (1995). Validity of psychological assessment: validation of inferences from persons' responses and performances as scientific inquiry into score meaning. American Psychologist, 50(9), 741-749.

Nehring, A. (2014). Wissenschaftliche Denk- und Arbeitsweisen im Fach Chemie. Eine kompetenzorientierte Modell- und Testentwicklung für den Bereich der Erkenntnisgewinnung. Berlin: Logos.

Neumann, K., Kauertz, A., Lau, A., Notarp, H., \& Fischer, H.E. (2007). Die Modellierung physikalischer Kompetenz und ihrer Entwicklung. Zeitschrift für Didaktik der Naturwissenschaften, 13, 101-121.

Neumann, K., Viering, T., Boone, W. J., \& Fischer, H.E. (2013). Towards a learning progression of energy. Journal of Research in Science Teaching, 50(2), 162-188.

Neumann, K., Viering, T., \& Fischer, H.E. (2010). Die Entwicklung physikalischer Kompetenz am Beispiel des Energiekonzepts. Zeitschrift für Didaktik der Naturwissenschaften, 16, 285-298.

NGSS Lead States (2013). Next Generation Science Standards: for states, by states. Washington: National Academies.

NRC (2012). A framework for K-12 science education. Practices, crosscutting concepts, and core ideas. Washington: National Academies.

OECD (2006). Assessing scientific, reading and mathematical literacy. A framework for PISA 2006. Paris: OECD.

OECD (2017). PISA 2015 assessment and analytical framework. Science, reading, mathematic, financial literacy and collaborative problem solving. Paris: OECD.
Parchmann, I. (2010). Kompetenzmodellierung in den Naturwissenschaften. Vielfalt ist wertvoll, aber nicht ohne ein gemeinsames Fundament. Review. In E. Klieme, D. Leutner \& M. Kenk (Hrsg.), Kompetenzmodellierung. Zwischenbilanz des DFG-Schwerpunktprogramms und Perspektiven des Forschungsansatzes (Bd. 56, S. 135-142). Weinheim: Beltz.

Ramseier, E., Labudde, P., \& Adamina, M. (2011). Validierung des Kompetenzmodells HarmoS Naturwissenschaften. Fazite und Defizite. Zeitschrift für Didaktik der Naturwissenschaften, 17, 7-33.

Renkl, A. (2012). Modellierung von Kompetenzen oder von interindividuellen Kompetenzunterschieden. Psychologische Rundschau, 63(1), 50-53.

Ropohl, M., Walpuski, M., \& Sumfleth, E. (2015). Welches Aufgabenformat ist das richtige? - Empirischer Vergleich zweier Aufgabenformate zur standardbasierten Kompetenzmessung. Zeitschrift für Didaktik der Naturwissenschaften, 21, 1-15.

Rost, J., Walter, O., Carstensen, C.H., Senkbeil, M., \& Prenzel, M. (2005). Der nationale Naturwissenschaftstest PISA 2003. Der mathematische und naturwissenschaftliche Unterricht, 58(4), 196-204.

Schecker, H., \& Parchmann, I. (2006). Modellierung naturwissenschaftlicher Kompetenz. Zeitschrift für Didaktik der Naturwissenschaften, 12, 45-66.

Schecker, H., Neumann, K., Theyßen, H., Eickhorst, B., \& Dickmann, M. (2016). Stufen experimenteller Kompetenz. Zeitschrift für Didaktik der Naturwissenschaften, 22, 197-213.

Schwichow, M., \& Nehring, A. (2018). Variablenkontrolle beim Experimentieren in Biologie, Chemie und Physik: Höhere Kompetenzausprägungen bei der Anwendung der Variablenkontrollstrategie durch höheres Fachwissen? Empirische Belege aus zwei Studien. Zeitschrift für Didaktik der Naturwissenschaften, 24, 217-233.

Upmeier zu Belzen, A., \& Krüger, D. (2010). Modellkompetenz im Biologieunterricht. Zeitschrift für Didaktik der Naturwissenschaften, 16, 41-57.

Vorholzer, A., Aufschnaiter, C. v., \& Kirschner, S. (2016). Entwicklung und Erprobung eines Tests zur Erfassung des Verständnisses experimenteller Denk- und Arbeitsweisen. Zeitschrift für Didaktik der Naturwissenschaften, 22, 25-41.

Weinert, F.E. (2000). Lehren und Lernen für die Zukunft. Ansprüche an das Lernen in der Schule. Pädagogische Nachrichten Rheinland-Pfalz, 2, 1-16.

Weinert, F.E. (2001). Vergleichende Leistungsmessung in Schulen eine umstrittene Selbstverständlichkeit. In F.E. Weinert (Hrsg.), Leistungsmessungen in Schulen (S. 17-31). Weinheim: Beltz.

Wellnitz, N., \& Mayer, J. (2013). Erkenntnismethoden in der Biologie - Entwicklung und Evaluation eines Kompetenzmodells. Zeitschrift für Didaktik der Naturwissenschaften, 19, 335-345.

Wellnitz, N., Fischer, H.E., Kauertz, A., Mayer, J., Neumann, I., Pant, H. A., et al. (2012). Evaluation der Bildungsstandards - eine fächerübergreifende Testkonzeption für den Kompetenzbereich Erkenntnisgewinnung. Zeitschrift für Didaktik der Naturwissenschaften, 18, 261-291.

Woitkowski, D., \& Riese, J. (2017). Kriterienorientierte Konstruktion eines Kompetenzniveaumodells im physikalischen Fachwissen. Zeitschrift für Didaktik der Naturwissenschaften, 23, 39-52.

Ziepprecht, K., Schwanewedel, J., Heitmann, P., Jansen, M., Fischer, H.E., Kauertz, A., et al. (2017). Modellierung naturwissenschaftlicher Kommunikationskompetenz - ein fächerübergreifendes Modell zur Evaluation der Bildungsstandards. Zeitschrift für Didaktik der Naturwissenschaften, 23, 113-125. 